University of Louisville

ThinkIR: The University of Louisville's Institutional Repository

Electronic Theses and Dissertations

$12-2004$

\title{
The analysis of missing data in public use survey databases : a survey of statistical methods.
}

Ping Xu 1975-

University of Louisville

Follow this and additional works at: https://ir.library.louisville.edu/etd

\section{Recommended Citation}

Xu, Ping 1975-, "The analysis of missing data in public use survey databases : a survey of statistical methods." (2004). Electronic Theses and Dissertations. Paper 1603.

https://doi.org/10.18297/etd/1603

This Master's Thesis is brought to you for free and open access by ThinkIR: The University of Louisville's Institutional Repository. It has been accepted for inclusion in Electronic Theses and Dissertations by an authorized administrator of ThinkIR: The University of Louisville's Institutional Repository. This title appears here courtesy of the author, who has retained all other copyrights. For more information, please contact thinkir@louisville.edu. 
THE ANALYISIS OF MISSING DATA IN PUBLIC USE SURVEY DATABASES:

A SURVEY OF STATISTICAL METHODS

\author{
By \\ Ping Xu \\ M.S., Capital University of Medical Sciences, China, 2001
}

\begin{abstract}
A Thesis
Submitted to the Faculty of the Graduate School of the University of Louisville in Partial Fulfillment of the Requirements for the Degree of

Master of Science in Public Health

Department of Bioinformatics and Biostatistics

University of Louisville

Louisville, Kentucky
\end{abstract}

December 2004 
THE ANALYSIS OF MISSING DATA IN PUBLIC USE SURVEY DATABASES:

A SURVEY OF STATISTICAL METHODS

\author{
By \\ Ping $\mathrm{Xu}$ \\ M.S., Capital University of Medical Sciences, China, 2001
}

A Thesis Approved on

November 19, 2004

by the following Thesis Committee:

Thesis Director 


\section{ACKNOWLEDGEMENTS}

There are many people that I would like to thank, but with the limited space it will be hard to mention them all. I would like to thank my advisor, Dr. Tonya M. Smoot, for the knowledge and insight she passed on to me, and for her guidance throughout all phases of this project. I also thank Dr. Steven McCabe and Dr. William Rising for taking the time to be a part of my thesis defense committee.

I thank my husband, Yan Ke, one of the most important people in my life, for sharing my happiness and sadness, and for his encouragement when I was frustrated. I thank my parents for being a source of support for me through the years.

Finally I would like to say thank you to all of my friends who helped me throughout the years. 


\title{
ABSTRACT \\ THE ANALYSIS OF MISSING DATA IN PUBLIC USE SURVEY DATABASES: A SURVEY OF STATISTICAL METHODS
}

\author{
Ping Xu
}

November 20, 2004

Missing data is very common in survey research. However, currently few guidelines exist with regard to the diagnosis and remedy to missing data in survey research. The goal of the thesis was to investigate properties and effects of three selected missing data handling techniques (listwise deletion, hot deck imputation, and multiple imputation) via a simulation study, and apply the three methods to address the missing race problem in a real data set extracted from the National Hospital Discharge Survey.

The results of this study showed that multiple imputation and hot deck imputation procedures provided more reliable parameter estimates than did listwise deletion. A similar outcome was observed with respect to the standard errors of the parameter estimates, with the multiple imputation and hot deck imputation producing parameter estimates with smaller standard errors. Multiple imputation outperformed the hot deck imputation by using larger significant levels for variables with missing data and reflecting the uncertainty with missing values.

In summary, our study showed that employing an appropriate imputation technique to handling missing data in public use surveys is better than ignoring it. 


\section{TABLE OF CONTENTS}

PAGE

ACKNOWLEDGEMENTS $\ldots \ldots \ldots \ldots \ldots \ldots \ldots \ldots \ldots \ldots$ iii

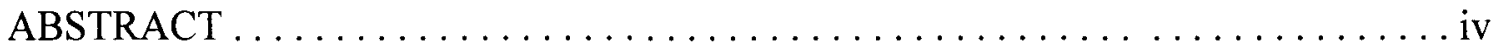



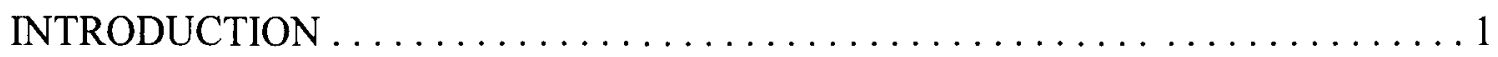

Impact of Missing Data in Surveys $\ldots \ldots \ldots \ldots \ldots \ldots \ldots \ldots \ldots \ldots \ldots \ldots \ldots \ldots$

Approaches to Handling Missing Data in Surveys . ................ 2

Missing Data in the National Hospital Discharge Survey . . . . . . . . . . . 4

Purpose of the Study . . . . . . . . . . . . . . . . . . . . . . . . 5

BACKGROUND AND REVIEW OF THE LITERATURE $\ldots \ldots \ldots \ldots \ldots \ldots \ldots 7$

The Problem of Missing Data in Survey Analysis . . . . . . . . . . . . . .

Types of Non-Response in Surveys $\ldots \ldots \ldots \ldots \ldots \ldots \ldots \ldots \ldots \ldots$

Item Non-Response in Surveys $\ldots \ldots \ldots \ldots \ldots \ldots \ldots \ldots \ldots \ldots$

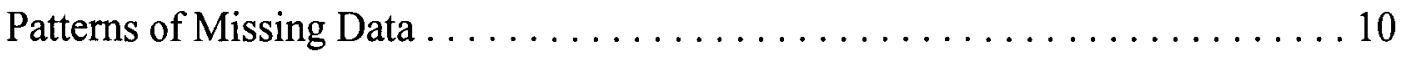

Mechanisms of Missing Data $\ldots \ldots \ldots \ldots \ldots \ldots \ldots \ldots \ldots \ldots \ldots \ldots \ldots$

Approaches to Handling Missing Data . . . . . . . . . . . . . . . . . . 14

The Problem of Missing Race in the NHDS $\ldots \ldots \ldots \ldots \ldots \ldots \ldots \ldots \ldots \ldots$

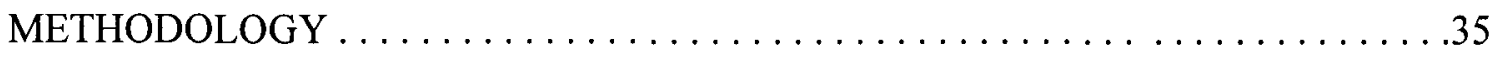

The Data ............................................

Research Question and Statistical Modeling $\ldots \ldots \ldots \ldots \ldots \ldots \ldots \ldots . \ldots \ldots$

Tests for Determining the Missing Data Mechanism .................38

Imputation Methods . . . . . . . . . . . . . . . . . . . . . . . 39 
Simulation of Missing Data . . . . . . . . . . . . . . . . . . . . 42

Statistical Analysis and Testing $\ldots \ldots \ldots \ldots \ldots \ldots \ldots \ldots \ldots \ldots \ldots \ldots$

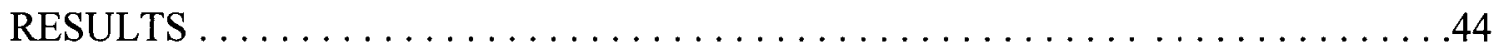

Results for Simulation Study . . . . . . . . . . . . . . . . . . . 44

Multiple Imputation for Missing Race $\ldots \ldots \ldots \ldots \ldots \ldots \ldots \ldots \ldots \ldots$

Descriptive Statistics of the Original and the Imputed Race ... . . . . . . . 48

Results of Logistic Regression Analyses

in Simulation study . . . . . . . . . . . . . . . . . . . 48

Results for the 2001 Gastric Bypass Data Set .................. 50

Testing the Randomness of Missing Race Data . . . . . . . . . . . 51

Descriptive Statistics of the Imputed Race Data . . . . . . . . . . . . . . .52

Comparison of Results of Logistic Regression Analyses . . . . . . . . . . . . 53

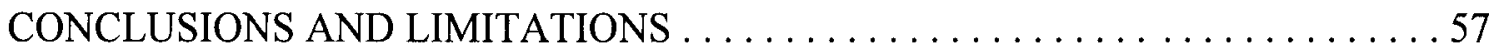

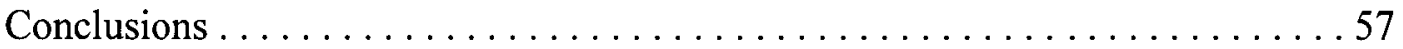

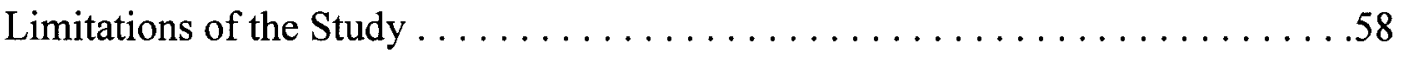

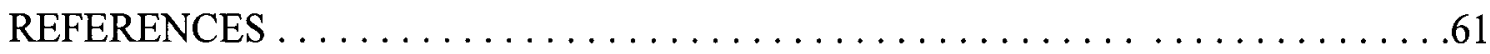

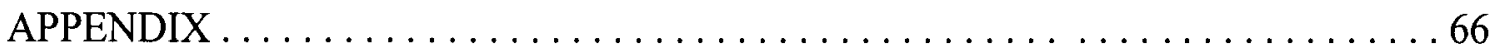

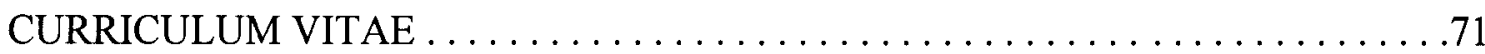




\section{LIST OF TABLES}

TABLE

PAGE

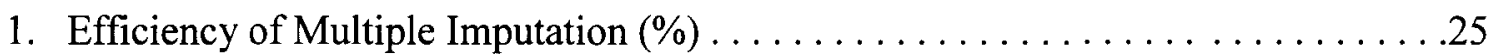

2. Comparison of the Original Race with the 5 Imputed

Race Values for One of the Cases. . . . . . . . . . . . . . 47

3. Distribution of Original and Imputed Race in Simulated Data .............48

4. Logistic Regression Results of the Simulated Data . . . . . . . . . . . . . . . . . 49

5. Comparison of Demographic Characteristics and CCI Between

Respondent with Complete Information and Respondents

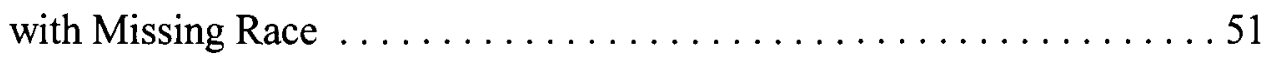

6. Distribution of the Observed and Imputed Race (\%) $\ldots \ldots \ldots \ldots \ldots \ldots . . \ldots \ldots$

7. Logistic Regression Analysis of Gastric Bypass Data with

Listwise Deletion.............................. 53

8. Logistic Regression Analysis of Gastric Bypass Data with

Hot Deck Imputation . . . . . . . . . . . . . . . . . . . 54

9. Logistic Regression Analysis of Gastric Bypass Data with

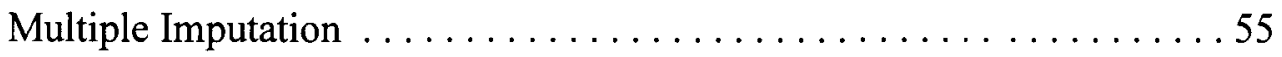




\section{INTRODUCTION}

Missing data are not uncommon in survey research in both social and health sciences. Many large public use survey databases suffer from different degrees of missing information on one or more key variables, which has always been a great concern to researchers. The problem of missing data in the surveys sometimes poses serious problems for researchers because nearly all standard statistical methods presume that every case observation has information on all the variables to be included in the analysis. Analysis ignoring missing data may result in misleading conclusions drawn from a research study and limit the generalizability of the research findings (Brick and Kalton, 1996). Given the prevalence and importance of missing data, researchers must be prepared to address the problems that may arise when data are missing. However, there are very few guidelines with regard to the diagnosis and remedy of such problematic missing data (Hair, Anderson and Black, 1998).

\section{Impact of Missing Data in Surveys}

When data are missing, the central statistical issues are power and bias (Fayers, Curran and Machin, 1998). Power reduction is one concern when missing data exist in a study. The power of a study is the statistical ability to reject the null hypothesis when it is actually false. Deletion of cases with missing values leads to a possible loss of power due to the reduced number of observations, which could prevent the researchers from rejecting the null hypothesis when it is indeed false. The other serious concern about 
missing data is that it has the tendency to introduce bias into a study (Becker and Powers, 2001; Rubin, 1987). The non-respondents might be systematically different from those who responded completely, thus the completely observed cases that remain will be unrepresentative of the population for which the inference is usually intended. If the researcher chooses to draw conclusions based solely on those who responded, the conclusion could be biased (Schafer, 1997). To avoid bias and power reduction, one approach that is frequently used by researchers is the imputation of missing data (Downey and King, 1998).

\section{Approaches to Handling Missing Data in Surveys}

When faced with missing data, a researcher has two primary options for dealing with this situation. First, he can ignore the missing data, and utilize only the complete cases in the statistical analysis. The second option is to find a way to replace the missing values with values from similar units in the dataset or with predicted values obtained from a model. These methods are also known as the imputation methods (Downey and King, 1998).

In studies where there is a relatively large amount of missing data, the researchers must either gather additional observations or find a remedy for the missing data in the original sample (Hair and Anderson, 1998). Most researchers agree that finding a remedy for missing data is a more practical solution than obtaining additional observations. Public use databases present a challenge. These databases are collected from national surveys and cover a wide range of areas, for instance, surveys of hospital usage, nutrition status, and other measures. Public use surveys are conducted using populations as a basis 
for sampling. Thus, it is impossible for researchers to gather additional observations in these types of data. Unfortunately, few guidelines exist pertaining to the remedy of missing data (Hair and Anderson, 1998).

The history of the development of statistical procedures for missing data can be divided into three periods (Schafer, 1997). In the first period, the 1970's, most widely applied methods dealing with missing data were ad hoc methods, which included listwise deletion, mean substitution, the simple hot-deck method, and various regression based methods. They were easy to implement, yet have the potential to produce biased results. In the second period, the 1980's, likelihood based estimation procedures using the Expectation-Maximization (EM) algorithm began to appear (Little and Rubin, 1987). These methods were generally superior to the ad hoc methods in that they are statistically efficient and produce parameter estimates with acceptable standard errors. Even though these methods are model specific and can be difficult to implement, they are viewed as breakthroughs in the history of missing data methods. The third period, the 1990's, was characterized by the introduction of multiple imputation methods, Markov Chain Monte Carlo (MCMC), and Bayesian methods, to overcome the limitations of single imputation methods such as mean substitution, hot deck imputation, regression based imputation methods, and likelihood based imputation methods (Schafer, 1997). Even though the multiple imputation method represents the latest effort by methodologists to deal with missing data, it has not been widely adopted in survey research (Schafer, 1997).

In the United States, there are several national population based surveys conducted on a regular basis. The investigation and comparison of approaches to 
handling missing data on one such survey, the National Hospital Discharge Survey, is the focus of this research.

\section{Missing Data in the National Hospital Discharge Survey}

The National Hospital Discharge Survey (NHDS) is an ongoing survey conducted by the National Center for Health Statistics. The NHDS collects medical and demographic information, and is an annual probability sample of discharges from nonfederal, short-stay (an average length of stay of less than 30 days), non-institutional hospitals in the United States. The NHDS data serve as a basis for information on the utilization of the nation's hospitals and the nature and treatment of illness among the hospitalized population (CDC, http://www.cdc.gov/nchs).

There are two types of non-responses found in the NHDS data. One type is the unit non-response, which occurs when no information is collected on a sample unit. The issue of the unit non-response in the NHDS data is routinely handled by the use of adjusting the sampling weights. The other type is item non-response, which occurs when the absence of data is only limited to some variables or items.

The NHDS collects information on hospital discharges that contain, among other data, patient characteristics (age, sex, and race), patient disposition at discharge, expected source of payment for hospitalization, up to seven discharge diagnosis codes and up to four procedure codes based on International Classification of Diseases, Ninth Revision, Clinical Modification (ICD-9-CM) codes, hospital size by number of beds, and geographic region (Gillum BS and Graves EJ, 1998). In total, the number of items collected in the NHDS has ranged from 30 to 32 over the many years the survey has been in existence. Among the items collected, seven variables have different degrees of 
missingness. Most of the item non-response rates are low except for two items with high non-response rate, race and marital status. Currently, missing values of age and sex in the NHDS are routinely imputed by a hot deck method that maintains the known age or sex distribution of records within the same 3-digit level of first-listed ICD-9-CM diagnostic code. However, there is generally no attempt made to impute the missing values of other items such as race (Gillum BS and Graves EJ, 1998). Race data from the NHDS has become increasingly incomplete in recent years. From 1982 through 1991, discharges with race not stated ranged from 8.9 to 17.8 percent of the total discharges (Kozak LJ, 1995). Then, from 1992 to 2001, discharges with missing race ranged from 19.8 to 27.9 percent of all the discharges in the NHDS. Researchers are faced with the problem of missing data when using the NHDS data, in particular when examining racial differences in patterns of hospital use. These may reflect differences in access to care or in the distribution of health problems.

\section{Purpose of the Study}

Although many different methods have been proposed for handling missing data in surveys, only a few have gained widespread popularity. Unfortunately, there is no clear consensus as to which method is generally preferred. The method of choice for a particular survey may depend upon particular circumstances including the reason for "missingness" or the mechanism for "missingness", the type of the survey data, and the availability of computer software. The purposes of the current study are to:

(1) Review and compare some commonly used methods for handling missing data in surveys. 
(2) Investigate properties and effects of three selected missing data handling techniques: listwise deletion, single imputation (hot deck), and multiple imputation via a simulation study based on the complete cases from the gastric bypass data set.

(3) Apply the three selected methods (listwise deletion, hot deck imputation, and multiple imputation) to address the missing race problem in the gastric bypass data from the 2001 NHDS.

Information and conclusions regarding to the similarities and discrepancies among these missing data handling techniques will be very useful to researchers who are struggling with missing data problems in analyzing large public use surveys, such as the NHDS. 


\section{BACKGROUND AND REVIEW OF THE LITERATURE}

This chapter reviews why missing data are a problem in survey analysis and what can be done when facing with missing data in surveys. In addition, this chapter also discusses the types of non-response in surveys, mechanisms of missing data, the patterns of missing data, and the possible approaches for handling missing data. Finally, this chapter discusses the problem of missing race in the NHDS data and how missing race problem has been addressed in previous studies using NHDS data.

\section{The Problem of Missing Data in Survey Analysis}

Missing data are universally problematic in survey research, with substantial amounts of missing data frequently appearing (Little and Rubin, 1987). In surveys, specific individuals may tend to not respond to certain questions. Participants inadvertently skip some questions, may not have the required information at hand, or choose not to respond (Patrician, 2002). Missing data are problematic for a number of reasons. The most serious concern is that missing data can introduce bias into estimates derived from a statistical model (Becker and Walstad, 1990). For example, it is possible that due to some unobserved underlying factors the non-respondents might be systematically different when compared with those who respond completely. Thus, the remaining sample is no longer random or representative of the population from which it was drawn. If the researcher chose to draw their conclusions based only on those who 
respond, the conclusions could be biased (Schafer, 1997).

Missing data also result in a loss of information and statistical power (Anderson and Basilevsky, 1983). The elimination of subjects with missing values on one or more variables from the statistical analysis decreases the statistical power due to reduced sample size. Another problem with missing data is that they make common statistical methods inappropriate or difficult to apply since most statistical procedures rely on complete-data methods of analysis (Rubin, 1987). For example, multivariate statistical methods, as they are programmed into some commonly used commercial statistical software such as SAS and SPSS, are applicable to complete data sets by default.

The seriousness of the above problems depends in part on how many data are missing. There is no clear rule regarding how many are too many missing data (Kline, 1998). Cohen (1983) suggested that $5 \%$ to $10 \%$ missing data on a variable may be considered small, while $40 \%$ missing data on a variable may be considered to be high (Raymond and Roberts, 1987). Although the impact of a small number of missing data may be less noticeable and contribute less statistical bias than larger proportions, potential bias is inherent whenever data are missing (Kline, 1998).

\section{Types of Non-response in Surveys}

In surveys, it is almost certain that some level of non-response will occur. Missing survey data can be classified into two main types. One type is total (or unit) nonresponse, which occurs when no information is collected on an entire sample unit. Total non- response often results from refusals to participate in the survey, non-contacts (notat-homes), and other reasons such as a language barriers, deafness, or being too ill to 
participate. The other type of non-response is item (or partial) non-response, which occurs when the absence of data is limited to only some variables. Item non-response may arise because a respondent refuses to answer an item on the grounds that it is too sensitive, does not know the answer to an item, gives an answer that is inconsistent with answers to other items and hence is deleted in editing, or because the interviewer fails to ask the question or record the answer (Brick and Kalton, 1996). For example, if a hospital is the unit of analysis and the nurse-executive at each hospital is the respondent, those who fail to return the survey would be unit nonresponsive. If, however, the nurseexecutive completes the survey except for several items, this would be a case of item nonresponse (Patrician P.A. 2002). In surveys, weighting methods are commonly used to compensate for unit non-response in which respondents are assigned greater weight in the analysis in order to represent the non-respondents, while the usual form of compensation for item non-response is imputation (Little and Rubin, 2002). The goal in both cases is to produce a dataset from which approximately unbiased parameter estimates can be derived during the process of hypothesis testing and data analysis. In most public use sample survey data, sampling weights based on probability of selection and adjusted for unit nonresponse and other factors are attached to each respondent record. This thesis, however, addresses only item non-response in surveys.

\section{$\underline{\text { Item Non-Reponses in Surveys }}$}

There are few guidelines in the literature pertaining to the remedy of missing data (Hair and Anderson, 1998) and, currently there is no generally preferred method for handling missing data (Curran and Molenberghs, 1998). When faced with missing data, 
the researcher can either ignore the missing data or use imputation techniques to handle missing data (Downey and King, 1998). Imputation methods make it possible to analyze the data as if it were complete (Musil and Warner, 2002). The objective of imputation is to employ known relationships that can be identified in the valid values of the sample, to assist in representing or estimating the replacements for missing values (Hair, Anderson and Black, 1998). There are different forms of imputation methods available to deal with item non-response for survey analysis. Some common methods of imputation used in surveys include deductive imputation, mean (median) imputation, Hot Deck imputation, Cold Deck imputation, regression imputation, stochastic regression imputaion, multiple imputation, and composite imputation methods (Musil and Warner, 2002). Each of these

methods has relative advantages and disadvantages. This study will explore the impact of both complete case analysis (listwise deletion) and two selected imputation methods (hot deck imputation and multiple imputation) on the results and inferences in statistical analysis on the gastric bypass data from the 2001 NHDS.

\section{Patterns of Missing Data}

Missing data occur to varying degrees and in various patterns (Cohen and Cohen, 1983). The impact of missing data on the validity of research findings depends on the proportion of missing data, the mechanisms that led to missing data, and the patterns of missing data (Tabachnick and Fridell, 2001). For a $\mathrm{n} \times \mathrm{p}$ data matrix ( $\mathrm{n}$ cases for $\mathrm{p}$ variables) with missing data, it is very useful to distinguish the missing data pattern, which describes which values are observed in the data matrix and which values are missing. There are 3 different patterns of missing data: the univariate pattern, the 
monotone pattern, and the arbitrary pattern. The missing data pattern is a univariate pattern when missingness is confined to a single variable. The missing data pattern is a monotonic pattern when the variables can be arranged so that $Y_{j+1}, \ldots, Y_{k}$ are missing for cases where $Y_{j}$ is missing, for all $\mathrm{J}=1, \ldots, \mathrm{K}-1$, and the ordering of variables is important. An example of monotonic pattern missing data would be attrition in longitudinal studies. Longitudinal studies collect information on a set of cases repeatedly over time, and a common missing data problem is attrition, where subjects drop out prior to the end of the study and do not return. Thus, all the data following the attrition will be unavailable. The missing data pattern is an arbitrary or general pattern, when missing data occur in a haphazard pattern and ordering of variables is unimportant. Methods for handling monotone missing data can be easier than methods for general patterns (Little and Rubin, 2002).

\section{Mechanisms of Missing Data}

To determine how to handle missing data, one must identify the reason for "missingness" or mechanism for missingness. There are three categories of missing data in relation to randomness: Missing completely at random (MCAR); missing at random (MAR); and not missing at random (NMAR) or non-ignorable missing data (Little and Rubin, 2002).

1. Missing Completely at Random (MCAR):

The missing data for a random variable Y are "Missing Completely At Random" if the probability of having a missing value for $\mathrm{Y}$ is not related to the value of $\mathrm{Y}$ itself or to any other variables in the data set (Allison, 2002). For example, missing income is 
MCAR if the probability of income being missing is not related to the value of income or to any other variables in the data set (Allison, 2002). Under the MCAR condition, missing data can be treated as a random sub-sample of the potentially complete data, and the missing data mechanism capturing the reasons for missing data can be ignored for sampling-based and likelihood-based inferences (Little and Rubin, 1987). MCAR is a very stringent assumption and it is rarely encountered in the research arena (Mills, 2001). Whether data are MCAR can be verified partly by comparing the non-responders to responders on all other variables. However, it would be nearly impossible to determine whether the probability of missing data was a result of the value of the variable itself (Allison, 2002).

2. Missing at Random

Data can be missing at random, a less restrictive notion than MCAR. Data are "Missing at Random" if the probability of missing data on the random variable $\mathrm{Y}$ is not dependent on the value of $Y$ itself but may depend on the values of other variables in the dataset (Allison, 2002). For example, the MAR assumption would be satisfied if the probability of missing data on income depended on a person's marital status, but within each marital status category, the probability of missing income was unrelated to income. The MAR assumption is impossible to test directly because there is no way of knowing the values of the missing data (Allison, 2002).

If the data are MCAR or MAR, and the parameters $\theta$ of the data model and the parameters $\phi$ of the model for the missing data indicators are distinct or unrelated, then the missing data mechanism is said to be ignorable. Ignorability basically means that there is no need to model the missing data mechanism as part of the estimation process 
(Allison, 2002; Schafer, 1997). Generally, it would be safe to assume that partially incomplete data from subjects who completed research assessment are ignorable missing data (Allison, 2002). When the missing data mechanism is ignorable, it is appropriate to impute values of the missing data. However, when the assumption of ignorable missing data is not met, imputation is usually not appropriate (Rubin, 1987).

3. Not Missing at Random (NMAR) or Nonignorable Missing Data

NMAR occurs when the probability of missingness depends on the missing values themselves (Little and Rubin, 2002; Rubin, 1976). This is also called the nonignorable missing data. For example, if high income households are less likely to report their income even after adjusting for other variables, then the probability of missing income is nonignorable (Allison, 2002). Nonignorable missing data have systematic, nonrandom factors underlying the occurrence of the missing values that are not apparent.

Nonignorable missing data are the most problematic because they affect generalizability of research findings, and may potentially bias parameter estimates. Neither single nor multiple imputation can be used for this type of data. More complicated methods are required for nonignorable missing data, and the missing data mechanism must be specified by the researcher and incorporated into the data analysis in order to produce unbiased parameter estimates (Allison, 2002).

Identifying the underlying patterns (nonrandom factors) that contribute to the occurrence of missing data is important because these influence how missing data should be handled. Too often a remedy is applied without an assessment of the missing data mechanism (Hair and Anderson, 1998). Testing the hypothesis of whether or not the missing data are MCAR is quite simple. There are two common methods to test for the 
randomness of missing data. One method is the two-sample $t$ tests between the missing observations and some other variables. If a variable $\mathrm{x}$ has missing data, groups are defined by having or not having a missing value on the variable $\mathrm{x}$. An independent twosample $t$ test can be used to compare mean differences between the two subgroups on other continuous variables in the data set. Statistically significant differences between the subgroup means indicate that missing data on the variable $\mathrm{x}$ are not operating in a random manner (not MCAR). Correspondingly, the Chi-square tests can be used to test the differences between the two subgroups on other categorical variables in the data set. The other method is the Pearson product-moment correlations. If the variable $\mathrm{x}$ is correlated with other variables in the data set, low correlation coefficients will reflect relatively more randomness and MCAR data, whereas higher statistically significant correlation coefficients will indicate the non-randomness associated with MAR. There is no rule of thumb to determine the cutoff point for a sufficiently high correlation that indicates nonrandomness, but statistical significance is one indicator of non-random data (Musil CM and Warner CB, 2002; Huisman, 1999). However, there is no comparable test available to test whether or not the data are MAR or NMAR (Curran and Bacchi, 1998).

\section{Approaches to Handling Missing Data}

There are two primary options for handling missing data: complete case analysis and imputation methods. These approaches are discussed in the following section.

\section{Listwise Deletion (Complete Case Analysis) — the simplest approach:}

Listwise deletion, also known as complete case analysis, is perhaps the simplest and the most popular method for dealing with incomplete data. Listwise deletion assumes 
incomplete cases are similar to complete cases with respect to the values of the variable with missing observations and of all the other variables. In this approach, all units or cases that contain any missing value for a relevant variable in the particular analysis are deleted. There are two obvious advantages to listwise deletion: (1) it can be used for any statistical analysis, from structural equation modeling to log-linear analysis; (2) no specific computational methods are required (Allison, 2002). Although this approach is easy to understand and to implement, it may result in a loss of power due to small sample size when the extent of missing data is high, a loss of generalizability of the results due to the loss of potentially important information, and potentially substantial biases if the data are not missing completely at random (Little and Rubin, 2002). Many researchers suggest that the listwise deletion method is best suited for instances in which the extent of missing data is small, the sample size large enough to allow for the deletion of the cases with missing data and the relationships in the data string so as not to be affected by any missing data process (Troxel and Fairclough, 1998).

\section{Creation of the Category "Unknown":}

This technique fills in the missing values with an additional category "unknown" so that complete data methods of statistical analysis can be used. However, interpretation of the findings of the statistical analysis could be confusing because of this artificial juxtaposition of measured levels of a particular variable and the use of a category code to earmark missing data (Levy PS and Lemeshow S, 1991). For these reasons, this method is rarely used in survey analysis. 


\section{Imputation Approaches:}

Imputation is defined as a process of estimating the missing values based on valid values of other variables and/or cases in the sample (Hair and Anderson). There are different forms of imputation methods available to deal with item non-response in survey analysis, and some are mathematically and computationally difficult to apply. There are two types of imputation methods used in surveys: single imputation and multiple imputation.

In single imputation, each missing value is replaced with a single value. Some frequently used single imputation methods include deductive imputation, mean (mode) imputation, regression imputation, and hot-deck imputation. Each of these methods has strengths and weaknesses.

Deductive imputation is applicable when a missing value can be deducted from responses to other variables. For example, a person aged under 16 years may be imputed to be single. As this example indicates, deductive imputation assumes a high degree of certainty about the missing values. In this sense, deductive imputation is often considered to be editing rather than imputation (Brick JM and Kalton G, 1996).

Mean imputation involves substituting the overall sample mean of a variable for each missing observation of that particular variable (Kline, 1998). Mean imputation is a simple and conservative approach because the sample mean does not change, but it disregards each subject's patterns of scores on other variables in the data set (Tabchnic BG and Fidell LS, 2001). However, mean substitution has many statistical pitfalls. According to Little and Rubin (1987), the limitations include: sample size is overestimated; variance is underestimated; correlations are negatively biased; and the 
distribution of new values is an incorrect representation of the population values because the shape of the distribution is distorted by adding values equal to the mean. The bias introduced into the population variance, correlation, and variable distribution depends on the amount of missing data and on the actual value that are missing. Little and Rubin's recommendation is to never use the mean substitution method.

Regression imputation imputes missing values with predicted values derived from the least square regression equation based on variables in the data set that contain no missing values. Variables with missing data are treated as dependent variables and are predicted by all of the variables having complete data (Little and Rubin, 2002; Musil and Warner, 2002). Regression imputation has the advantage of yielding an unbiased point estimate of the missing value and using relationships already existing in the sample as the basis of prediction. It preserves cases with missing data and maintains the sample size. Regression imputation also has several disadvantages. Because the imputed values are always perfectly predicted from the regression model, the correlations and covariances are inevitably inflated. Moreover, applying the regression imputation to multivariate data sets can be difficult when more than one variable has missing values (Donner, 1982).

Another method is the hot-deck imputation, in which the data are stratified and sorted by key covariates, and each missing datum is randomly replaced from that of another record in the same stratum (Little and Rubin, 2002).

As stated earlier, the mean substitution, regression imputation, and hot deck imputation handle the missing data problem by imputing missing values once, thus they are referred to as single imputation methods. Single imputation methods have the advantage of being simple to implement for any type of variable. However, single 
imputation does not reflect the uncertainty about the predictions of the unknown missing values, and the resulting estimated variances and the standard errors of the parameter estimates will be biased toward zero. Furthermore, the sample size behaves as if it were overstated, confidence intervals for estimated parameters are too narrow, and Type I error are too high (Little and Rubin, 1987; Rubin, 1987).

To overcome the limitations of methods that fail to take into account the uncertainty associated with imputed values, Rubin developed the multiple imputation method in the 1980's. Multiple imputation replaces each missing value with a set of plausible values drawn from the distribution of the data to represent the uncertainty about the right value to impute (Allison, 2002).

This research focuses on the complete case analysis and two of the imputation techniques: the hot deck imputation and the multiple imputation. Hot deck imputation was chosen because it has been widely used by the Census Bureau for over 40 years (Reilly and Pepe, 1997), and it is also routinely used by many large public use survey data collected by the National Center for Health Statistics. For example, beginning with the 1996 NHDS, a hot deck method for missing values of age and sex has been used that maintains the known age or sex distribution of records within the same 3-digit level of first-listed diagnostic code. Multiple imputation was chosen since it can correct the limitations of single imputation, and it is one of the most attractive methods for general purpose handling of missing data in multivariate analysis. Multiple imputation has been utilized to handle missing data in some large NCHS health surveys such as the National Health and Nutritional Examination Survey (NHANES III). Multiple imputation has also been successfully used in the National Health Interview Survey (NHIS) to impute single 
race categories for those who have given multiple race responses (Schenker $\mathrm{N}$ and Parker JD, 2003). A recent Medline search (search terms: NHDS, missing data, multiple imputation) showed that in the last decade (1994-2004), there were no published studies in which multiple imputation methods were applied to handling missing data in the subgroup data analysis extracted from the NHDS. The Details about hot deck imputation and multiple imputation techniques are described below.

(1) Hot Deck Imputation

The term "hot deck" literally refers to the deck of matching computer cards for the donors available for a non-respondent (Little and Rubin, 2002), and in the imputation procedure, the "deck" refers to the deck of responses of respondents with completed items from which the researcher may select a value (Curran and Molenberghs, 1998). The value may be selected simply at random or by employing elaborate schemes. With most hot-deck procedures, individuals with missing values are matched with those having similar values in a set of other variables and the known value is imputed into the missing cell, for example, selecting a random value from only those respondents who have similar characteristics, such as gender, age or treatment groups (Little and Rubin, 2002; Ford, 1983). Little and Rubin (2002) also listed several other ways that a missing value may be selected in the hot deck procedure. In the nearest neighbor hot deck, a distance measure is defined between subjects, based on the values of covariates, and then the imputed values are chosen from responding subjects close to the subject with the missing value. With the sequential hot deck method, a set of imputation classes is defined, and for each imputation class a computer location is created in which a value of the variable to be imputed (y) is to be stored. The survey records are then considered sequentially 
throughout the data file. If a record has a value for $y$, that value will be stored in the location for the record's imputation class, replacing the value currently residing in that location. If a record has a missing y value, it will be assigned the y value currently stored in the location for its imputation class (Little and Rubin, 2002; Brick and Kalton, 1996). Hot deck imputation has a long history of use. The United States Census Bureau has used the hot-deck imputation since 1960 (Little and Rubin, 2002). The hot deck procedure has been used to impute missing values by many governmental agencies. Data sets repaired by hot deck imputation strategies are routinely accepted as representing accurate samples of the study population. It has also been used by many public use health surveys conducted by NCHS, by the Current Population Surveys, and by the Survey of Income and Program Samples, etc.

The primary concerns when using a hot deck approach are selecting a valid "characteristic set" to identify potential donors who will provide a reasonable distribution of potential outcomes for the missing values; and ensuring that the "characteristic set" allows the donor pool to remain large enough to allow for reasonable variance among imputed replacement values. Obtaining a balance between these two goals is often difficult to achieve, as it is hard to arrive at the right combination of homogeneity of donor and recipient pools and variation in potential outcomes (McNally JW, 1997). Hot deck imputation has several advantages over other approaches (Ford, 1983). Because a hot deck approach selects imputed values at random from a donor pool of complete cases, it introduces variation into the analysis set consistent with the range of possible values seen in the complete data set. As a result, there are fewer tendencies towards the mean of the sample. The other main advantage of this non-parametric 
technique is that it does not require strong distribution assumptions or careful modeling to develop selection criteria for imputing a value (Perez A and Rodolfo JD, 2002). The hot deck approach frees the analyst of most parametric assumptions, as the ultimate goal is to randomly choose from the observed variation found within a pool of donors selected on the basis of a small set of uniform characteristics. While hot decking should not be thought of as a superior model based approaches to imputation and data replacement, it does have the advantages of being relatively easy to use and of providing consistent and reliable results if the appropriate characteristic set is selected (McNally JW, 1997). The disadvantage of the method is that there are no criteria to guide the selection of the donor set of complete cases, because it is very hard to define "similar". The definition may vary from one researcher to another, creating uncertainty to the results. Another disadvantage of hot deck imputation is the extensive programming that is required to implement this technique (Perez A and Rodolfo JD, 2002).

(2) Multiple Imputation

Multiple imputation was first proposed by Rubin nearly twenty years ago (Rubin, 1978). However, this method had remained largely unknown and unused by non-experts until the late 1990's, when a variety of new simulation methods, known collectively as Markov Chain Monte Carlo, were adapted and implemented for the purpose of multiple imputation (Schafer, 1997). As defined by one of its leading proponents, "multiple imputation is the technique that replaces each missing or deficient value with two or more acceptable values representing a distribution of possibilities" (Rubin, 1987). Multiply imputed data reflect the uncertainty contained in the imputation process in a way not possible with singly imputed data (Rubin, 1987). Rubin's multiple imputation (MI) was 
further explicated by Schafer in 1997 and by Allison in 2002. Multiple imputation does not attempt to estimate each missing value through a simulated value but rather to represent a random sample of the missing values. MI is a predictive approach to handling missing data in multivariate analysis. It blends both classical and Bayesian statistical techniques and relies on specific iterative algorisms to create several imputations. MI aims to create plausible imputations of the missing values, to accurately reflect uncertainty, and to preserve important data relationships and aspects of the data distribution (Freedman and Wolf, 1995; Schafer, 1997).

\section{Advantages and Disadvantages of Multiple Imputation (MI)}

The advantages of MI build on the benefits of single imputations. MI allows use of complete data methods for data analysis. MI can accommodate any model and any type of variables. Moreover, MI reflects the missing data uncertainty. MI incorporates appropriate random error into the imputation process and makes it possible to get approximately unbiased estimates of all model parameters. No deterministic imputation method can do this in general settings. Because repeated estimates are used, MI produces more reasonable estimates of standard errors than single imputation methods. Single imputation methods don't allow for the additional random error introduced by imputation (Allison PD, 2001; Patrician PA, 2002).

MI also has some disadvantages. First, $\mathrm{MI}$ is computationally intensive, and there is limited software available for MI. However, these disadvantages lessen and pose fewer problems as technology advances (Patrician PA, 2002). Second, MI requires a probability model for the complete data. Third, MI does not produce a unique result. Because randomness is preserved in the MI process, each data set imputed will yield slightly 
different estimates and standard errors. Therefore, the reproducibility of exact results may be problematic (Rubin, 1987; Schafer, 1997). Finally, MI has been criticized because of novices' reactions to it. Rubin (1996) said that initially MI was thought to be unacceptable because it used simulation and added random noise to the data. MI was once considered by many to be a form of "statistical alchemy" (Schafer, 1999). But Schafer (1999) claims that MI accurately represents the observed information; it does not simply conjure data.

\section{Assumptions and Requirements of Multiple Imputation}

Certain requirements must be met for MI to have desirable properties. First, the data must be missing at random (MAR), which means the probability of missing data on a particular variable $Y$ can depend on other observed variables, but not on $Y$ itself. Second, the imputation model should be compatible (at least approximately) with the model used for analysis (Allison, 2002). Rubin (1987) termed this a "proper" imputation model. This model should include all variables in the desired analysis as well as other variables predictive of the missing information. Third, the algorithm used to generate imputed values must be "correct". Allison compared the results of two different algorithms for producing multiple imputations. The first algorithm considered only the variables associated with the missingness of the data (also known as propensity score method) and the second included other variables and their associations. Allison's results clearly support Rubin's contention that good imputation methods use all information related to missing cases (Allison, 2000; Patrician, 2002).

Although the requirements and assumptions described above are very important for multiple imputation, Allison (2000) presented simulation results showing that 
multiple imputation is robust to model violations, whereas some alternatives (listwise deletion, propensity scoring) are not. King et al. (2001) reported simulation results showing that multiple imputation works well, even in situations where the assumptions of MI are violated.

\section{The Procedure of Multiple Imputation}

The procedure of multiple imputation is quite straightforward. First, impute the missing values using an appropriate model that incorporates random variation. Second, do this $m$ times (usually 3-5 times), producing $m$ "complete" data sets. Third, perform the desired analysis on each data set using standard complete data methods. Finally, average the values of the parameter estimates across the $m$ samples to produce a single point estimate; calculate the standard errors by specific computational formula that incorporates the number of imputations and the variance among the parameter estimates (Rubin, 1987). The three steps of multiple imputation are illustrated in Figure 1.

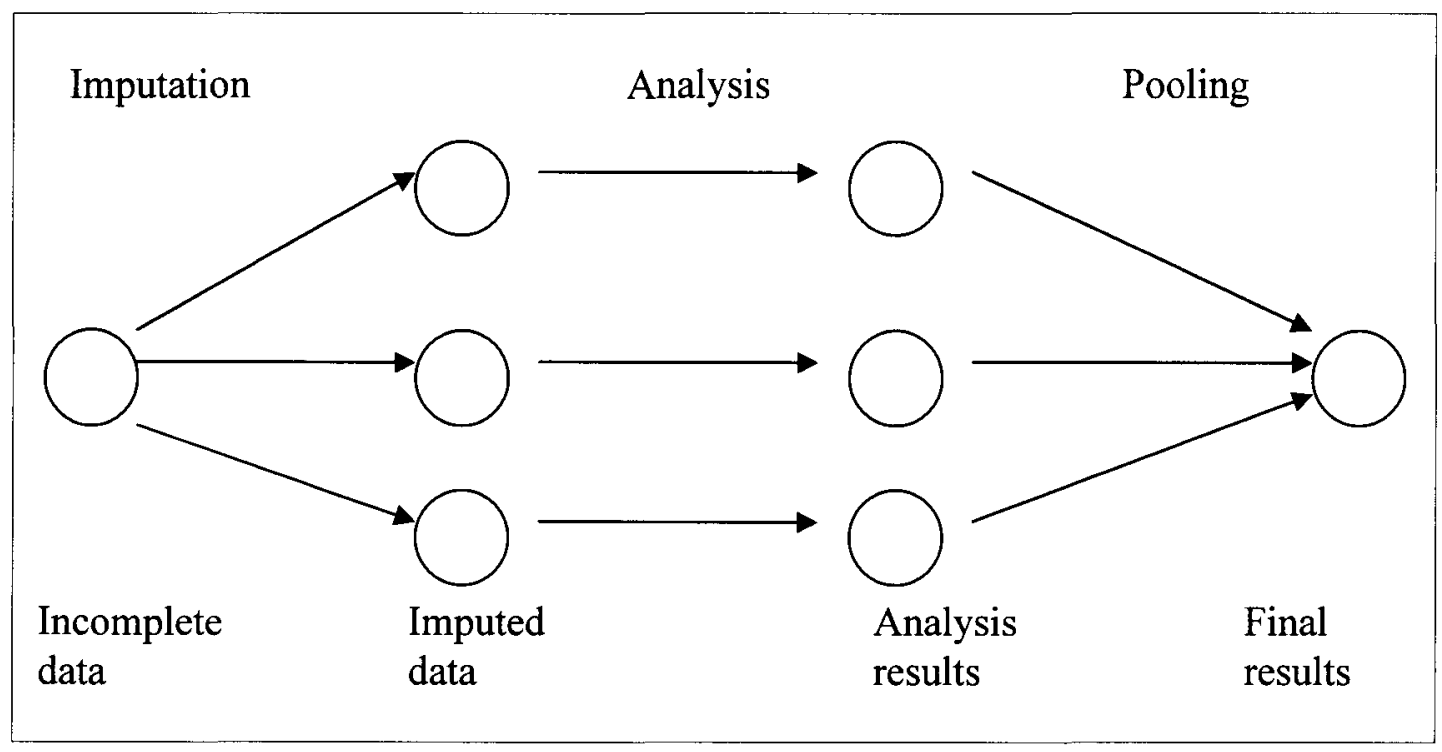

Figure 1. Three steps of multiple imputation, adapted from Multiple Imputation Online (Van Buuren and Oudshoorn, 2001) 
The relative efficiency of an estimate based on the finite $m$ imputations, rather than using an infinite number for the fully efficient imputation, is approximately $(1+\gamma / \mathrm{m})^{-1}$, where $\gamma$ is the fraction of missing information (Rubin, 1987). Table 1 lists the relationships of fractions of missing data with the number of imputations for efficiency of recovery of the true parameter. In many applications, 3 to 5 imputations are sufficient to obtain excellent results (Schafer and Olsen, 1998).

Table 1

Efficiency of multiple imputation (\%)

\begin{tabular}{llllll}
\hline \multicolumn{5}{c}{$\gamma$} \\
\hline $\mathrm{m}$ & 0.1 & 0.3 & 0.5 & 0.7 & 0.9 \\
\hline 3 & 97 & 91 & 86 & 81 & 77 \\
5 & 98 & 94 & 91 & 88 & 85 \\
10 & 99 & 97 & 95 & 93 & 92 \\
20 & 100 & 99 & 98 & 97 & 96 \\
\hline
\end{tabular}

The pooled estimates are computed using formulas given by Rubin (1987). For an imputed data set $i$, let the regression coefficient estimate for an explanatory variable be denoted as $b_{i}$ and its variance be $V_{i}, i=1, \ldots m$. The final pooled estimate for the regression coefficient is the average of all $b_{i}$ 's:

$$
\bar{b}=\frac{1}{m} \sum_{i=1}^{m} \mathrm{~b}_{\mathrm{i}}
$$


The within imputation variance of these point estimates is given by:

$\mathrm{W}=\frac{1}{m} \sum_{i=1}^{m} \mathrm{~V}_{\mathbf{i}}$

The between imputation variance of these point estimates is given by:

$\mathrm{B}=\frac{1}{m-1} \sum_{i=1}^{m}\left(\mathrm{~b}_{\mathrm{i}}-\bar{b}\right)^{2}$

The total variance associated with the pooled estimate $\bar{b}$ is:

$\mathrm{T}=\mathrm{W}+\left(1+\frac{1}{m}\right) \mathrm{B}$

The confidence interval of the estimate can be calculated using the approximation:

$\bar{b} \pm \mathrm{t}_{d f} \sqrt{T}$

where $t_{d f}$ denotes a quantile of Student's t-distribution with degrees of freedom

$d f=(\mathrm{m}-1)\left(1+\frac{m W}{(m+1) B}\right)^{2}$

$\mathrm{P}$-values for testing the null hypothesis $\mathrm{b}=0$ may be obtained by comparing the ratio

$\bar{b} / \sqrt{T}$ to the same t-distribution.

Let Q denotes a population quantity of interest. Rubin (1987) also shows that an estimate of the fraction of missing information about the population quantity $Q$ is:

$\gamma=\frac{r+2 /(d f+3)}{r+1}$ 
where $r=\frac{(1+1 / m) B}{W}$ is the relative increase in variance due to non-response. Both $\gamma$ and $r$ are useful diagnostic statistics, revealing how strongly the estimation of Q may be influenced by missing data.

Imputation Models for Multiple Imputation

To perform multiple imputation, an imputation model must be selected to generate the imputations. The most popular model for MI is the multivariate normal model. The multivariate normal model implies that all variables in the model have normal distributions and each variable can be represented as a linear function of all the other variables, together with a normal, homoscedastic error term. Most real datasets depart substantially from these assumptions, but in practice the multivariate normal models seem to do a good job of imputation even when some of the variables have distributions that are manifestly not normal (Allison, 2002; Schafer, 1997). Simulations show that multiple imputation is fairly robust to model misspecification (Schafer, 1997). It is a completely innocuous assumption for those variables that have no missing data because nothing is being imputed for them. For those variables that do have missing data, normalizing transformation can greatly improve the quality of imputations (Allison, 2002; Schafer, 1997).

For situations in which all variables in the imputation process are categorical, a more attractive model is the unrestricted multinomial model (which has a parameter for every cell in the contingency table) or a log-linear model that allows restrictions on the multinomial parameters. Furthermore, a general location model is available for data containing both categorical and quantitative variables. This method presumes that the categorical variables have a multinomial distribution, possibly with log-linear restrictions 
on the parameters. Within each cell of the contingency table created by the categorical variables, the quantitative variables are assumed to have a multivariate normal distribution. The means of these variables are allowed to vary across cells, but the covariance matrix is assumed to be constant (Allison, 2002; Schafer 1997). Schafer (1997) showed how these models also can be used as the basis for data augmentation to produce multiple imputation, and he developed the freeware programs called CAT and MIX to implement the methods (http://www.stat.psu.edu/ jls/). However, both CAT and MIX are available only as libraries to the S-PLUS statistical package and R. In both cases, the underlying models potentially have many more parameters than the multivariate normal model. As a result, effective use of these methods typically requires more knowledge and input from the person performing the imputation, together with a larger sample size to achieve stable estimates (Allison, 2002).

Although the methods and computer programs (CAT and MIX) were designed strictly for data sets with only categorical variables, as well as for data sets with mixtures of categorical and normally distributed variables, these methods are typically much more difficult to use and sometimes fail to produce reliable results if the sample size not large enough (Allison, 2002). Many users will do just as well by applying the normal methods with some minor alterations. Dichotomous variables, such as sex, are usually represented by dummy variables with values of 0 and 1 , and they can simply be imputed just like any other variable. Then the imputed values can be rounded to 0 or 1 . Values with more than two categories are usually represented with sets of dummy variables, and can be imputed just like the dichotomous variable (Allison, 2002; Schafer 1997). As a routine method for 
handling missing data, multiple imputation under the multivariate normal model is probably the best that is currently available.

\section{Imputation Mechanisms for Multiple Imputation}

There are three common methods used for imputing missing values depending on the missing data pattern. For monotone missing data patterns, either a parametric regression method that assumes multivariate normality or a nonparametric method that used propensity scores is appropriate. For an arbitrary missing data pattern, a Markov chain Monte Carlo (MCMC) method that assumes multivariate normality can be used (Allison, 2000; SAS Institute Inc, 1999; Schafer, 1997).

(a) Regression Method:

A data set with variables $Y_{1}, Y_{2}, \ldots, Y_{p}$ (in that order) is said to have a monotone missing data pattern when the event that a variable $Y_{j}$ is observed for a particular individual implies that all previous variables $Y_{k}, k<j$, are also observed for that individual. In the regression method, a regression model is fitted for each variable with missing values, with the previous non-missing variables as covariates. Based on the resulting model, a new regression model is then fitted and is used to impute the missing values for each variable (Rubin, 1987).

(b) Propensity Score Method:

A propensity score is generally defined as the conditional probability of assignment to a particular category given a vector of observed covariates (Rosenbaum and Rubin, 1983). In this method, a propensity score is generated for each variable with missing values to indicate the probability of that observation being missing. The observations are then grouped based on these propensity scores, and an approximate 
Bayesian bootstrap imputation is applied to each group. For example, in group $\mathrm{k}$, suppose that $Y_{o b s}$ denotes the $n_{l}$ observations with nonmissing $Y_{j}$. The approximate Bayesian bootstrap imputation first draws $n_{1}$ observations randomly with replacement from $Y_{\text {obs }}$ to create a new data set $\mathrm{Y}_{\mathrm{obs}}{ }^{*}$. This is a nonparametric analogue of drawing parameters from the posterior predictive distribution of the parameters. For each missing case, the process then randomly draws one value with replacement from the random sample $\mathrm{Y}_{\mathrm{obs}}{ }^{*}$ and used the observed value as the imputed value (Lavori, and Dawson, 1995). The propensity score method only uses information from covariates that are associated with whether the imputed variable values are missing. It does not use correlations among variables. It is effective for inferences about the distributions of individual imputed variables, but it is not appropriate for analyses involving relationship among variables (Allison, 2000; Yang, 2000).

(c) Markov Chain Monte Carlo (MCMC) Method

A Markov Chain is a sequence of random variables in which the distribution of the each observation depends only on the value of the previous one (Yang, 2000). The MCMC method is the most widely used method to produce multiple imputation, and is recommended by Allison (2002) and Schafer (1997). It is suitable for both arbitrary and monotone patterns of missing data. The multiple imputation using MCMC method assumes data are from a multivariate normal distribution.

In the MCMC procedure, after the imputation model is selected, the next step is to generate the maximum likelihood estimates of the means and covariance matrix using the EM algorithm. The EM algorithm formalizes a relatively old ad hoc idea for handling missing data. The first step is to replace missing values by estimated values; second, 
estimate the parameters; third, re-estimate the missing values assuming the new parameter estimates are correct; and finally re-estimate parameters, and so forth, iterating until convergence (Little and Rubin, 1987). Each iteration of the EM consists of two steps. The expectation step (E-step) calculates the conditional expectation of the missing data given the observed data and current estimated parameters, and then substitutes these expectations for the missing data. The maximization step (M-step) finds the parameter estimates to maximize the complete data log-likelihood from the E step. After this, the expectation step resumes and calculates new imputations using the new mean and correlation. The two steps are iterated until the iterations converge and yield the maximum likelihood estimates. Converge occurs when the estimates barely change from one iteration to the next (Allison, 2002). EM's rate of convergence is determined by the rates of missing data in the dataset. If there were no missing values, then convergence would be immediate; if large amounts of data are missing, the convergence will require many iterations. One way to monitor the convergence of EM is to examine the log likelihood function and confirm that it increases at each iteration (Schafer, 1997). In the next step, the data augmentation algorithm (DA), a member of the class of Markov Chain Monte Carlo algorithms (Schafer, 1997), uses the initial values obtained from the EM algorithm to generate the multiple data sets. Data augmentation is a general method for finding posterior distributions that has become increasingly popular in Bayesian statistics. The posterior distribution is a Bayesian probability distribution created from both a prior and a conditional distribution (Allison, 2002). Like the EM algorithm, DA is an iterative process that alternatively fills in the missing data and makes inferences about the unknown parameters. However, DA does this in a stochastic or 
random fashion. DA first performs a random imputation of missing data under assumed values of the parameters, and then randomly draws new parameters form a Bayesian posterior distribution based on the observed and imputed data. The procedure of alternately simulating missing data and parameters creates a Markov chain that eventually stabilizes or converges in distribution. The distribution of the parameters stabilizes to a posterior distribution that averages over the missing data, and the distribution of the missing data stabilizes to a predictive distribution-the exact distribution that one needs to draw from to create proper multiple imputations (Schafer, 1997).

Assuming that the data are from a multivariate normal distribution, data augmentation is applied to Bayesian inference with missing data by repeating the following two steps. First, the imputation step (the I-step) simulates the missing data items by randomly selecting a value for each missing value from the available distribution of values. Next, the posterior step (the P-step) recomputes the mean vector and covariance matrix with the imputed estimates from the I-step, which is the posterior distribution. These two steps are iterated until the convergence of the joint posterior distribution of missing data and parameters (Schafer, 1997; Yang, 2000). The MCMC method was chosen for multiple imputation of missing race in this thesis.

\section{The Problem of Missing Race in the NHDS}

Race data in health surveys provide critical information to target and evaluate public health interventions, particularly for minority populations. One of the two national health objectives for 2010 is to eliminate health disparities among different segments of 
the population including racial disparities (U.S. Department of Health and Human Services, 2000). Currently, data from NHDS use the following race categories: White, Black, American Indian/Alaskan Native, Asian, Native Hawaiian, other, multiple race indicated, and not stated. Race data from the NHDS has become increasing incomplete in recent years. From 1982 to 1992 , discharges with race not stated ranged from $8.9 \%$ to $19.8 \%$ of total discharges in the NHDS, while from 1998 to 2002, discharges with missing race ranged from $22 \%$ to $29 \%$. There are several reasons responsible for the increasing underreporting of race in the NHDS. The primary reason is that a growing number of the hospitals that participated in the NHDS did not report race for any of their patients. Most of these hospitals used an automated data collection method that did not require an entry for patients race (Kozak LJ, 1995). Some other reasons include: state laws do not mandate collection of race data; confidentiality concerns discourage reporting of race and ethnicity; survey participation is voluntary and providers are not mandated to collect or report race data. In addition, race was not reported for the majority of patients that were identified as Hispanic (Kozak LJ, 1995).

Data from the NHDS have been used to examine the racial differences in patterns of hospital use that may reflect differences in access to care or in the distribution of health problems. Recent studies that have used race data from the NHDS have investigated a variety of topics, including stress urinary incontinence surgery (Waetjen LE and Subak LL, 2003), episiotomy use (Weber AM and Meyn L, 2002), invasive cardiac procedures (Giles WH and Anda RF, 1995), hysterectomy (Wilcox LS and Koonin LM, 1994), HIV (Kozak LJ and McCarthy E, 1993), stroke (Modan B and Wagener DK, 1992), children's asthma (Gergen PJ and Weiss KB, 1990), coronary 
arteriography and coronary bypass surgery (Ford E and Cooper R, 1989), and hip fractures (Rodriguez JG and Sattin RW, 1989), among others. At present, there is no ideal solution to eliminate the problem of missing race data in the NHDS. Most researchers exclude cases with missing race from their analysis, and some researchers use proportional adjustment, in which the discharges in the race-not-stated category are assigned to specific race category based on the distribution of the discharges where race is known. However, proportional adjustment is not appropriate when performing multivariate analysis. To date, imputation methods have not been utilized to solve the problem of missing race data in NHDS. Exploratory analyses of data from the survey suggest that imputation methods that use demographic and contextual covariate information to predict primary race can have advantages with respect to lower bias and improved variance estimation compared to other simpler methods (Schenker $\mathrm{N}$ and Parker JD, 2003). The purpose of this study was to apply three different missing data handling procedures (listwise deletion, hot deck imputation, and multiple imputation) to the subset of patients in the 2001 NHDS who underwent gastric bypass surgery for obesity to examine the similarities and discrepancies among them. 


\section{METHODOLOGY}

\section{The Data}

The Data used in this study were from the 2001 National Hospital Discharge Survey (NHDS). The National Hospital Discharge Survey (NHDS) is an ongoing survey conducted by the National Center for Health Statistics. The NHDS collects medical and demographic information, via an annual probability sample of discharges from nonfederal, short-stay (an average length of stay of less than 30 days), non-institutional hospitals in the United States. The NHDS sample includes with certainty the largest hospitals. The remaining sample of hospitals is based on a stratified, three-stage design. A detailed description of the sample design and data collection method of the NHDS has been published (Centers for Disease Control and Prevention, http://www.cdc.gov/nchs about/major/hdasd/nhds.htm). The NHDS data serve as a basis to provide more complete and precise information on the utilization of the nation's hospitals and on the nature and treatment of illness among the hospitalized population (CDC, http://www.cdc.gov/nchs).

The NHDS collects information on hospital discharges that contain, among other variables, patient characteristics (age, sex, and race), patient disposition at discharge, expected source of payment for hospitalization, up to seven discharge diagnosis codes and up to four procedure codes based on International Classification of Diseases, Ninth Revision, Clinical Modification (ICD-9-CM) codes, hospital size by number of beds, and geographic region (Gillum BS and Graves EJ, 1998). These data provide useful 
information that allows for the study of a variety of different diagnoses and procedures.

\section{$\underline{\text { Research Question and Statistical Modeling }}$}

The prevalence of obesity in the United States population continues to increase, making obesity a major public health concern (Mokdad AH and Bowman BA, 2000). Treatment options for obesity include various types of behavioral counseling, pharmacotherapy, and bariatric surgery. Of all these options, surgery has been shown to be the most efficacious (Mun EC and Blackburn GL, 2001). Currently the most commonly used bariatric surgery in the U.S. is the gastric bypass surgery (Mun EC and Blackburn GL, 2001). Several studies have found that the majority of patients undergoing bariatric procedures are women (Pope, 2002; Zizza, 2003). Researchers have also noted that male gender is a predictor of greater BMI before surgery and more comorbidities and complications for patients undergoing gastric bypass surgery (Rand CSW and Kuldau JM, 1993; Livingston EH and Huerta S, 2002). Zizza (2003) examined the gender differences in bariatric patients using the North Carolina Hospital Discharge Database, and found that male and female bariatric patients were different with respect to the number of comobidities, with more women having no comorbidities, and more men having at least one comorbidity. However, none of the above reports adjusted for other variables that may be related with the comorbidities in bariatric patients, such as age, race, and geographic region. This study intended to investigate the independent associations between age, sex, race, and the geographic region with the comorbidities for patients undergoing gastric bypass surgery. 
A population of patients undergoing gastric bypass surgery for the treatment of obesity was identified using ICD-9-CM diagnosis codes and procedure codes. Patients diagnosed with morbid obesity (278.01), unspecified obesity (278.00), obesity (278.0), and localized adiposity (278.1) were extracted from the 2001 NHDS data set if they had the accompanying procedure codes for high gastric bypass (44.31) or other gastroenterostomy bypass (44.39). For this analysis we only included patients 18 years of age and older. Patients who had diagnosis codes corresponding to abdominal neoplasm and other malignant neoplasm were excluded from consideration in the analysis (codes 150.0 through 159.9 , inclusive). The study sample consisted of 416 adult patients who underwent gastric bypass surgery for the treatment of obesity in 2001, with the mean age of $40.9 \pm 10.4$ years (mean $\pm \mathrm{sd}$ ). In the sample, there were 361 females and 55 males. $17.8 \%$ of the sample was from Northeast region, $40.1 \%$ was from Midwest region, $32.0 \%$ was from South region, and $10.1 \%$ was from West region. Five variables were extracted for our analysis data set: age, sex, race, geographic region, and a modification of the Charlson Comorbidity Index (CCI, $1=$ one or more comorbidities, $0=$ otherwise). The Charlson Comorbidity Index was used to measure the burden of comorbid disease in the sample (D'Hoore W and Sicotte C, 1996). This index weights specific secondary diagnoses based on their 1-year relative risk of mortality. These are then summed to achieve an index score for each patient that is a measure of the burden of comorbid disease. The diagnostic codes of the independent predictors used in the index are those for myocardial infarction, congestive heart failure, peripheral vascular disease, dementia, cerebrovascular disease, chronic pulmonary disease, connective tissue disease, ulcer disease, mild liver disease, hemiplegia, moderate to severe renal disease, diabetes, any 
tumor, leukemia, lymphoma, moderate to severe liver disease, and metastatic solid tumor (D'Hoore W and Sicotte C, 1996; Romano PS, and Roos LL, 1993). In this study, the Charlson Comorbidity Index was grouped into a dichotomous variable CCI: 0 (Charlson Comorbidity Index equals to 0 ) and 1 (Charlson Comorbidity Index $\geq 1$ ). In addition, a sampling weight variable was included to account for the survey's complex sample design. All the variables except for race were complete. Race data were missing for 137 out of 416 patients (approximately $32.9 \%$ ).

A multiple logistic regression model was fitted to the gastric bypass data from the 2001 NHDS using the dichotomous variable-the modified Charlson comorbidity index (CCI) as the dependent variable and age, sex, race, and geographic region as the explanatory variables.

\section{Tests for Determining the Missing Data Mechanism}

Prior to conducting any imputation and analyses, it must be ascertained whether or not the missing data process is missing completely at random. The demographic characteristics (age, sex race, geographic region) and Comorbidity Index of the respondents who had complete information on all of the five variables were compared to the demographic characteristics and Comorbidity Index of respondents who had missing race data. The continuous variable such as age was compared using the two sample t-test. Categorical variables such as sex were compared by Chi-squared tests. Significant differences between these two groups of respondents would indicate that the missing data mechanism is not MCAR. We assumed that the missing race was MAR in this study. 


\section{$\underline{\text { Imputation Methods }}$}

\section{$\underline{\text { Hot deck imputation }}$}

In the hot deck imputation, all the observations in the sample were partitioned into two groups: observations with complete race information and observations with missing race. The record providing the race value is called the donor, and the record with the missing race value is called the recipient. Imputation classes were established based on the values of the selected auxiliary variables sex, age group $(18-34,35-49,50-64, \geq 65)$, geographic region, and $\mathrm{CCI}$ to find a match of the donor and the recipient. Donors were selected by randomly sampling with replacement from the donor pool based on the recipient's imputation class, and then the recipient's missing race was replaced by the selected donor's race (McNally JW, 1997). These class imputations imply that persons with missing race data are a random sample of the persons in their class based on sex, age group, geographic region and CCI.

If there were imputation classes with recipients but without donors, then after the initially detailed matching of donors and recipients, the level of detail was collapsed where necessary to ensure that donors were found for all recipients. For example, in this data set, age group has four levels, but if no match can be found, it might be modified to be omitting the age group as a classification criterion.

After the hot deck imputations were completed, the multiple logistic regression was then performed on the imputed complete data set, using the dichotomous variableCharlson comorbidity index as the dependent variable and age, sex, race and geographic region as the explanatory variables. 


\section{Multiple imputation with the Markov Chain Monte Carlo (MCMC) method}

1. Step 1- Imputation

In this step, each missing value is replaced by a multiple number of imputations ( $\mathrm{m}$ imputations). Since the rate of missing race was about $30 \%$ in the gastric bypass data set, with " $\mathrm{m}=5$ " imputations, we would achieve $94 \%$ efficiency. Increasing the number to " $\mathrm{m}=10$ " raises the efficiency to $97 \%$, a rather slight gain for a doubling of computational effort. Therefore, in this study, we chose " $\mathrm{m}=5$ ".

The imputation model adopted in this study was the same logistic regression model that would be used subsequently to analyze the data, though these two models need not be identical. Multiple imputation with the MCMC method assumes the multivariate normal distribution, but in practice most real data may depart from this assumption. Schafer (1997) suggested that we may still be able to use the normal model to produce good-quality imputations even there are violations of assumptions in the underlying model. Simulations show that multiple imputation is fairly robust to model misspecification (Schafer, 1997). If there are missing values on the binary variables, they can be imputed under the normal model and rounded off to the nearest category. Values with more than two categories can be represented with sets of dummy variables, and then imputed just like the dichotomous variable (Allison, 2002).

For the imputation model used in this study, age and the binary variables sex and $\mathrm{CCI}$ can directly be included in the imputation model. The categorical variables race and geographic region were represented with 2 and 3 dummy variables respectively. After the imputation model was determined, the data matrix consisting of one dependent variable (CCI) and the four explanatory variables was submitted to PROC MI in SAS version 8.2 
to impute missing race data (race1 and race2). This was repeated 5 times. The convergence for the imputation process was checked by the time-series plot and the autocorrelation plot of the worst linear function (WLF). The worst linear function is a scalar function of parameters (means and covariance) that is the "worst" in the sense that it is the slowest to converge among parameters in the MCMC process. Hence, convergence of this function is evidence that other parameters are likely to converge as well (Schafer, 1997).

The other assumption of MCMC method was that missing data were missing at random (MAR). Because the primary reason for missing race in the NHDS was a growing number of hospitals that participated in the NHDS did not report race for any of their patients, the assumption of MAR for missing race was considered reasonable.

After the imputation, an additional variable "1- race1- race2" was calculated, and the final value of race was determined by the maximum value of race1, race2, and 1race1-race2 (Allison, 2002). In other words, if the imputed race1 is the maximum one, then race is "black"; if the imputed race2 is the maximum one, then the race is "other"; and if 1 - race 1 - race 2 is the maximum, then the race is "white".

2. Step 2 - Analysis

In the analysis step, each of the 5 complete data sets was analyzed by the standard multiple logistic regression model, using the dichotomous variable-Charlson comorbidity index as the dependent variable and age, sex, race and geographic region as the explanatory variables. 


\section{Step 3 - Pooling}

The last step of MI is to pool results of the 5 analyses to yield a final result. The procedure PROC MIANALYZE in SAS 8.2 was used. The pooled estimates are computed using formulas given by Rubin (1987) described in the previous chapter.

\section{Simulation of Missing Data}

It is often difficult to determine and compare the accuracy of different imputation methods, unless one is able to retrieve the missing data. A method must be devised to create a dataset that mimics the real life data, but where the true value of the missing data is known (Engels JM, Diehr P, 2003).

To form the simulated population with missing race data, respondents who had complete information on all variables from the gastric bypass patients in the 2001 NHDS comprised the dataset for the simulation study $(\mathrm{n}=279)$. By assigning a uniform random number to each of the 279 respondents, a moderate missing rate $25 \%$ of race data was made missing. The reason for choosing a $25 \%$ missing rate of race data is because this is the average missing rate of race data in the NHDS between 1998 and 2002. The resulting data set mirrored the actual patterns of the missing race in the sample. The complete case analysis, hot deck imputation, and multiple imputation were used on the simulated dataset to handle the problem of missing race.

Chi-square tests were used to compare the distributions of race after listwise deletion and imputation to the original distribution of race. The results of logistic regression analysis after listwise deletion or imputation were also compared to the known 
values from the original data set. The same research question and logistic regression model were used in the simulation study.

\section{Statistical Analysis and Testing}

Weighted methods were used for all statistical testing to account for the survey's sample design. Statistical significance was declared when the computed p-value was less than 0.05 . All statistical tests were two-sided and all analyses were performed using SAS version 8.2 for Windows software (SAS Inc., Cary, North Carolina). The code for all programs used in the study was provided in the Appendix. 


\section{RESULTS}

\section{Results for Simulation Studies}

The simulation studies were based on the 279 respondents who had complete information on all variables in the 2001 gastric bypass data set, and $25 \%$ of race data were randomly made missing. Then the listwise deletion, hot deck imputation, and multiple imputation method were used on the simulated dataset to handle the problem of missing race. The logistic regression model using the modified Charlson comorbidity index (CCI) as the dependant variable and age, sex, race, geographic region as the explanatory variables was fitted to the original data set with complete race data, the simulated data set with $25 \%$ missing race, and the imputed data sets respectively. The objective of the simulation study was to compare the results of the three missing data handling methods (listwise deletion, hot deck imputation, and multiple imputation) with the known values from the original dataset with complete information on all variables.

\section{Multiple Imputation for Missing Race}

The Markov Chain Monte Carlo (MCMC) method with multiple chains for the imputation process were implemented using the SAS procedure PROC MI. 200 burn-in iterations were completed before each imputation set was created. These 200 burn-in iterations were thought to be enough to make the iterations converge to a stationary distribution before the imputation process started filling in missing values. Stationary, in 
the normal case, means that the mean and variance of the distribution for the parameters being imputed are not changing over time (SAS Institute Inc, 1999). Since we do not have prior information about the mean and covariance estimates, a non-informative Jeffrey's prior was used for estimating the mean and covariance in the posterior step. This is the default prior implemented in SAS. The EM estimates were used as the initial estimates for MCMC. The convergence in the MCMC process was checked by the timeseries plot and the autocorrelation plot of the worst linear function (Figure 2 and Figure 3). The time-series plot in Figure 2 does not show any apparent long-term upward or downward trend for the worst linear function. In Figure 3, the dashed lines show approximate $95 \%$ confidence limit for autocorrelations. Although there is a positive autocorrelation in the initial lag for the worst linear function, this correlation died out very quickly, and there do not appear to be any other significant positive or negative autocorrelations after that. Therefore, the data augmentation (DA) algorithm can reasonably be said to have converged in the MCMC process. 


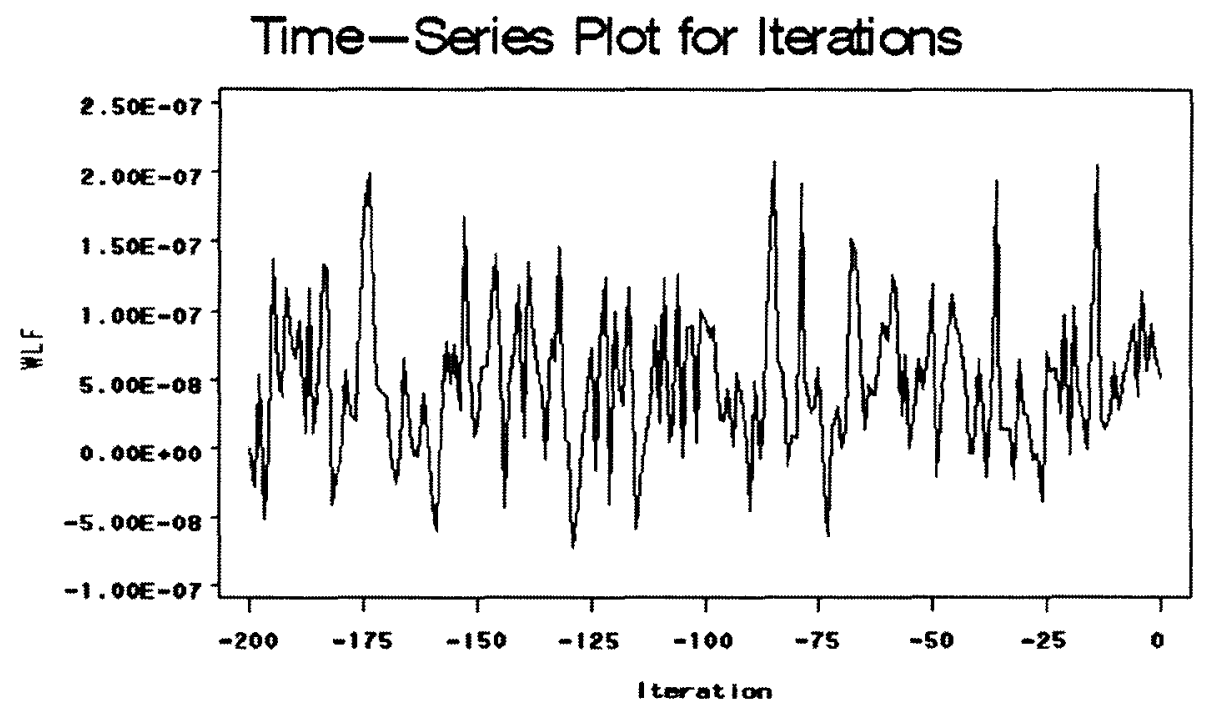

Figure 2. Time-Series plot of covariance for the worst linear function.

Autocorrelations with $95 \%$ Confidence Limits

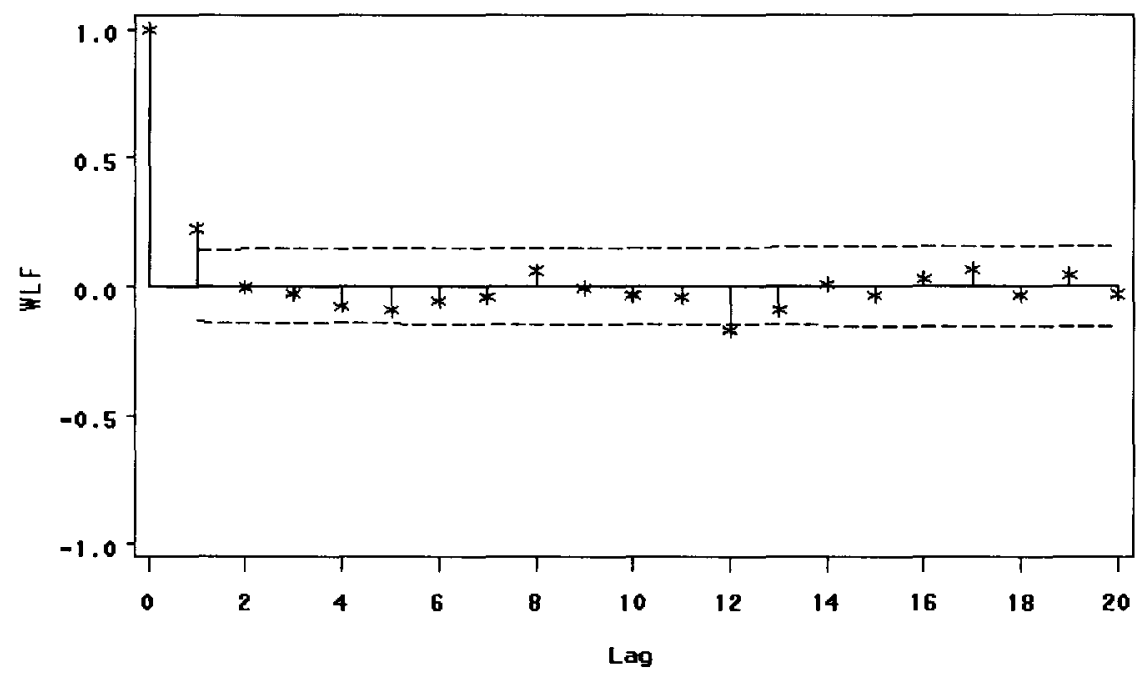

Figure 3. Autocorrelation plot of the worst linear function. 
Table 2 demonstrates the performance of the multiple imputation procedure by comparing the original race with the 5 imputed race values for one of the cases (ID=19). The original race of this case is white (race $=1$ ). Among the five imputed race values for this particular case, four imputed values indicate that the race is white, and one indicates that the race is black. The variability of the imputed values reflects the uncertainty associated with the missing values, which could not be accounted for by single imputation methods.

Table 2

Comparison of the original race with the 5 imputed race values for one of the cases

Original data for case $\mathrm{ID}=19$

$\begin{array}{ccccccccc}\mathrm{CCI} & \text { sex } & \text { age } & \text { geo1 } & \text { geo2 } & \text { geo3 } & \text { race1 } & \text { race2 } & \text { race } \\ 0 & 0 & 50 & 1 & 0 & 0 & 0 & 0 & 1\end{array}$

Data from the 5 imputed data sets for case ID $=19$

imp CCI sex age geo1 geo2 geo3 race1 race2 1-racel-race2 imputed race

$\begin{array}{lllllllllll}1 & 0 & 0 & 50 & 1 & 0 & 0 & 0.47 & 0.28 & 0.25 & 2 \\ 2 & 0 & 0 & 50 & 1 & 0 & 0 & 0.14 & 0.28 & 0.58 & 1 \\ 3 & 0 & 0 & 50 & 1 & 0 & 0 & 0.26 & 0.19 & 0.55 & 1 \\ 4 & 0 & 0 & 50 & 1 & 0 & 0 & 0.44 & 0.05 & 0.51 & 1 \\ 5 & 0 & 0 & 50 & 1 & 0 & 0 & 0.17 & 0.05 & 0.78 & 1\end{array}$


The imputed race values obtained by the hot deck and multiple imputations for the 70 cases with randomly made missing race were added to the 209 complete cases with intact race data. The distribution of race in the original data set and those in the imputed data sets are shown in Table 3. The results indicate that when compared with the race distribution in the original data set, all the three methods (listwise, hot deck, and multiple imputation) could maintain the distribution of the race data very well, though all of the three methods slightly overestimated the percentage of black race. Chi-square tests comparing the distributions of race in the imputed data sets with that in the original data set indicated that none of the imputed race distributions were significantly different from the race distribution in the original complete data set.

Table 3

Distribution of original and imputed race (\%) in simulated data

\begin{tabular}{lcccc}
\hline & $\begin{array}{c}\text { Original } \\
(\mathrm{n}=279)\end{array}$ & $\begin{array}{c}\text { Listwise Deletion } \\
(\mathrm{n}=209)\end{array}$ & $\begin{array}{c}\text { Hot Deck } \\
(\mathrm{n}=279)\end{array}$ & $\begin{array}{c}\text { Multiple Imputation } \\
(\mathrm{n}=279)\end{array}$ \\
\hline & & & & \\
White & 78.8 & 78.0 & 78.1 & 74.2 \\
Black & 15.8 & 16.3 & 17.2 & 19.6 \\
Other & 5.4 & 5.7 & 4.1 & 6.2 \\
\hline
\end{tabular}

Results of Logistic Regression Analyses with Simulated Data

The results of the logistic regression analysis with the original data set and in the imputed data sets are summarized in Table 4 . 
Table 4

Logistic regression results of the simulated data

\begin{tabular}{|c|c|c|c|c|}
\hline Method & $\begin{array}{l}\text { Original } \\
(\mathrm{n}=279)\end{array}$ & $\begin{array}{l}\text { Listwise } \\
(\mathrm{n}=209)\end{array}$ & $\begin{array}{c}\text { Hot Deck } \\
(\mathrm{n}=279)\end{array}$ & $\begin{array}{c}\text { MI } \\
(n=279)\end{array}$ \\
\hline \multicolumn{5}{|l|}{ Intercept } \\
\hline$\beta$ & -0.391 & -0.959 & -0.435 & -0.376 \\
\hline SE & 0.645 & 0.759 & 0.648 & 0.653 \\
\hline P-value & 0.544 & 0.206 & 0.502 & 0.566 \\
\hline \multicolumn{5}{|l|}{ Sex } \\
\hline$\beta$ & 1.074 & 1.485 & 1.076 & 1.061 \\
\hline $\mathrm{SE}$ & 0.383 & 0.430 & 0.382 & 0.383 \\
\hline P-value & 0.005 & 0.0005 & 0.005 & 0.006 \\
\hline \multicolumn{5}{|l|}{ Age } \\
\hline$\beta$ & 0.015 & 0.024 & 0.016 & 0.015 \\
\hline SE & 0.014 & 0.016 & 0.014 & 0.014 \\
\hline P-value & 0.254 & 0.138 & 0.227 & 0.260 \\
\hline \multicolumn{5}{|l|}{ Race (black) } \\
\hline$\beta$ & 0.434 & 0.247 & -0.478 & -0.016 \\
\hline $\mathrm{SE}$ & 0.356 & 0.439 & 0.398 & 0.356 \\
\hline $\mathrm{P}$-value & 0.223 & 0.574 & 0.230 & 0.965 \\
\hline \multicolumn{5}{|l|}{ Race (other) } \\
\hline$\beta$ & -1.429 & -1.914 & -1.305 & -0.693 \\
\hline SE & 1.179 & 1.731 & 1.201 & 1.152 \\
\hline P-value & 0.225 & 0.553 & 0.278 & 0.559 \\
\hline \multicolumn{5}{|c|}{ Geo (northeast) } \\
\hline B & -2.243 & -1.533 & -2.113 & -2.151 \\
\hline SE & 0.510 & 0.553 & 0.505 & 0.510 \\
\hline P-value & $<.0001$ & 0.006 & $<.0001$ & $<.0001$ \\
\hline \multicolumn{5}{|c|}{ Geo (midwest) } \\
\hline$\beta$ & -0.587 & -0.604 & -0.457 & -0.550 \\
\hline SE & 0.448 & 0.517 & 0.447 & 0.459 \\
\hline P-value & 0.189 & 0.243 & 0.307 & 0.232 \\
\hline \multicolumn{5}{|l|}{ Geo (south) } \\
\hline$\beta$ & -1.153 & -1.161 & -0.954 & -1.048 \\
\hline $\mathrm{SE}$ & 0.413 & 0.456 & 0.406 & 0.412 \\
\hline P-value & 0.005 & 0.011 & 0.019 & 0.012 \\
\hline
\end{tabular}

Note: Geo: geographic region

Table 4 shows that, compared with the logistic regression results from the original complete data, essentially all the three methods (listwise deletion, hot deck, and multiple imputation) found the same relationship between the dependant variable and the two 
explanatory variables sex and geographic region. However, there were important differences among these results. First, the parameter estimates, standard errors, and $\mathrm{p}$ values obtained by incorporating the two imputation methods, especially the multiple imputation, were generally much closer to the results in the original data than those generated by the listwise deletion method. Second, the standard errors of the parameter estimates obtained by using the multiple imputation and hot deck imputation were generally smaller than those obtained from the listwise deletion method. Third, the $\mathrm{p}$ values associated with the t-tests in the pooled logistic regression based on the multiple imputation were larger for race than those associated with the Wald's chi-square tests in the logistic regression based on the hot deck imputation.

These differences indicate that both multiple imputation and hot deck imputation provided more reliable parameter estimates than the listwise deletion, which were primarily due to the increased statistical power associated with the increased sample size, and their ability to preserve important data relationships and aspects of the data distribution (Freedman and Wolf, 1995). The increased significance levels for race after using the multiple imputation also illustrates that multiple imputation even did a better job than hot deck imputation by capturing the uncertainty associated with missing values and consequently leading to more valid statistical inferences in terms of both null hypothesis testing and interval estimation of the regression coefficients.

\section{Results for the 2001 Gastric Bypass Data Set}

The gastric bypass data set, extracted from the 2001 NHDS, contains 416 adult patients who underwent gastric bypass surgery for the treatment of obesity in 2001. In 
this data set, race was missing for 137 out of the 416 patients (about $32 \%$ ). The three missing data handling methods (listwise deletion, hot deck imputation, and multiple imputation) previously investigated in the simulation study were applied to this real data set to address the missing race problem.

\section{Testing the Randomness of Missing Race Data}

Prior to conducting any analyses, the randomness of missing data needs to be determined. All the observations in the sample were divided into respondents with missing race and respondents with complete information on all variables. Table 5 shows the results of the differences of demographic characteristics and the modified Charlson Comorbidity Index $(\mathrm{CCI})$ between respondents with missing race and respondents with complete information.

Table 5

Comparison of demographic characteristics and CCI between respondent with complete information and respondents with missing race.

\begin{tabular}{llll}
\hline & $\begin{array}{l}\text { Respondents with } \\
\text { complete information } \\
(\mathrm{n}=279)\end{array}$ & $\begin{array}{l}\text { Respondents with } \\
\text { missing race } \\
(\mathrm{n}=137)\end{array}$ & P-value \\
\hline Age (mean $\pm \mathrm{sd})$ & $40.6 \pm 10.3$ & $41.3 \pm 10.5$ & 0.49 \\
Sex (female, \%) & 88.2 & 83.9 & 0.29 \\
CCI $(\geq 1, \%)$ & 33.7 & 46.0 & $0.02^{*}$ \\
Geography (\%) & & & $<0.001^{*}$ \\
$\quad$ Northeast & 21.9 & 9.5 & \\
Midwest & 26.1 & 68.6 & \\
South & 45.2 & 5.1 & \\
West & 6.8 & 16.8 & \\
\hline
\end{tabular}

Note: CCI: modified Charlson Comorbidity Index

Respondents with missing race were not significantly different from respondents without missing race with respect to age and sex. However, compared with respondents 
having complete information on all variables, more respondents with missing race had one or more comorbidies $(\mathrm{CCI} \geq 1)(\mathrm{P}=0.02)$. Respondents with missing race were also significantly different from respondents without missing race with respect to the geographic regions. The significant differences between these two groups of respondents with respect to $\mathrm{CCI}$ and geography regions indicated that the missing race data mechanism was not missing completely at random (MCAR) but rather missing at random (MAR). Therefore, it would be very important to impute the missing race to preserve the relationships among the variables in the final analysis model.

\section{Descriptive Statistics of the Imputed Race Data}

The imputed race values obtained by the hot deck and multiple imputations for the 137 cases with missing race were added to the 297 complete cases with intact race data. The distributions of race in cases with complete race information and in all of the cases after imputation are shown in Table 6 . The results indicate that both the hot deck imputation and the multiple imputation were able to maintain the observed distribution of the race data very well. Chi-square tests comparing the distributions of race data in the imputed data sets with that in the observations with known race did not show any significant difference. 
Table 6

Distribution of the observed and imputed race (\%)

\begin{tabular}{lccc}
\hline & $\begin{array}{c}\text { Listwise Deletion } \\
(\mathrm{n}=279)\end{array}$ & $\begin{array}{c}\text { Hot Deck } \\
(\mathrm{n}=416)\end{array}$ & $\begin{array}{c}\text { Multiple Imputation } \\
(\mathrm{n}=416)\end{array}$ \\
\hline & & & \\
White & 78.8 & 80.7 & 75.6 \\
Black & 15.8 & 15.1 & 18.9 \\
Other & 5.4 & 4.2 & 5.5 \\
\hline
\end{tabular}

\section{Comparison of Results of Logistic Regression Analyses}

First, the logistic regression model using the modified Charlson comorbidity index $(\mathrm{CCI})$ as dependent variable and age, sex, race, geographic region as explanatory variables was fitted to the 279 observations with complete information. This was equivalent to treating the missing race data with the listwise deletion. Results were presented in Table 7.

Table 7

Logistic regression analysis of gastric bypass data with listwise deletion, $\mathrm{N}=279$

\begin{tabular}{lrccl}
\hline Parameter & \multicolumn{1}{c}{$\beta$} & $\mathrm{SE}$ & $\mathrm{P}$ & Odds Ratio( $\left.\mathrm{e}^{\mathrm{B}}\right)$ \\
\hline Intercept & -0.3912 & 0.6451 & 0.5442 &.-- \\
Sex & 1.0737 & 0.3831 & 0.0051 & 2.926 \\
Age & 0.0154 & 0.0135 & 0.2540 & 1.016 \\
Race (black) & 0.4335 & 0.3558 & 0.2231 & 1.543 \\
Race (other) & -1.4293 & 1.1789 & 0.2254 & 0.239 \\
Geo (Midwest) & -2.2431 & 0.5096 & $<.0001$ & 0.106 \\
Geo (Northeast) & -0.5873 & 0.4478 & 0.1896 & 0.556 \\
Geo (South) & -1.1526 & 0.4133 & 0.0053 & 0.316 \\
& & & & \\
\hline
\end{tabular}

According to this model, the log of the odds of having one or more comorbidities in gastric bypass patients was positively related with $\operatorname{sex}(\mathrm{p}=0.005)$, with male patients 
being 2.9 times more likely to have one or more comorbidities than female patients; The $\log$ of the odds of having one or more comorbidities was also related with geographic region. Compared with patients from the West region, patients from the Midwest and South were less likely to have one or more comorbidities $(p<0.01)$. Age and race were not significantly related with the log of the odds of having one or more comorbidities.

Next, the same logistic regression model was also fitted to the complete data with missing data replaced by imputed values from hot deck imputation. Table 8 shows the results of the logistic regression.

Table 8

Logistic regression analysis of gastric bypass data with hot deck imputaion, $\mathrm{N}=416$

\begin{tabular}{lrccc}
\hline Parameter & \multicolumn{1}{c}{$\beta$} & $\mathrm{SE}$ & $\mathrm{P}$ & Odds Ratio $^{\beta} \mathrm{e}^{\beta}$ \\
\hline Intercept & -0.8720 & 0.4767 & 0.0673 &.--- \\
Sex & 0.8044 & 0.3100 & 0.0095 & 2.235 \\
Age & 0.0238 & 0.0104 & 0.0226 & 1.024 \\
Race (black) & 0.4329 & 0.3098 & 0.1623 & 1.542 \\
Race (other) & -2.3117 & 1.0793 & 0.0322 & 0.099 \\
Geo (Midwest) & -1.5124 & 0.3296 & $<.0001$ & 0.220 \\
Geo (Northeast) & -0.5798 & 0.3213 & 0.0711 & 0.560 \\
Geo (South) & -0.9844 & 0.2936 & 0.0008 & 0.374 \\
& & & & \\
\hline
\end{tabular}

From Table 8, we can see that the log of the odds of having one or more comorbidities in gastric bypass patients was positively related with gender $(\mathrm{p}=0.009)$, with males being 2.2 times more likely to have one or more comorbidities than females; The log of the odds of having one or more comorbidities was also related with geographic regions $(\mathrm{p}<0.001)$. Age and race were also significantly related with the log of the odds of having one or more comorbidities in this model. For every 10 years increase in age, the risk of having one or more comorbidities increased $27 \%$. 
Finally, the same logistic regression model was fitted to each of the five complete data sets with missing data replaced by imputed values from multiple imputation respectively. By the PROC MIANALYZE, the results from the five logistic regression analyses were pooled into the final results (Table 9).

Table 9

Logistic regression analysis of gastric bypass data with multiple imputation, $\mathrm{N}=416$

\begin{tabular}{lcccc}
\hline Parameter & \multicolumn{1}{c}{$\beta$} & $\mathrm{SE}$ & $\mathrm{P}$ & ${\text { Odds Ratio }\left(\mathrm{e}^{\mathrm{B}}\right)}$ \\
\hline Intercept & -0.8649 & 0.4875 & 0.0769 &.-- \\
Sex & 0.8194 & 0.3097 & 0.0085 & 2.269 \\
Age & 0.0216 & 0.0103 & 0.0376 & 1.022 \\
Race (black) & 0.1763 & 0.3597 & 0.6298 & 1.193 \\
Race (other) & -0.7616 & 1.1756 & 0.5346 & 0.467 \\
Geo (Midwest) & -1.3780 & 0.3267 & $<.0001$ & 0.252 \\
Geo (Northeast) & -0.5106 & 0.3167 & 0.1077 & 0.600 \\
Geo (South) & -0.8389 & 0.2852 & 0.0035 & 0.432 \\
& & & & \\
\hline
\end{tabular}

According to Table 9, the log of the odds of having one or more comorbidities in gastric bypass patients was positively related with gender $(p=0.009)$, with males were 2.3 times more likely to have one or more comorbidities than females; The log of the odds of having one or more comorbidities was also related with geographic regions $(p<0.01)$. Age was also significantly related with the log of the odds of having one or more comorbidities in this model. For every 10 years increase in age, the risk of having one or more comorbidities increased $24 \%$. Race was not significantly related with the log of the odds of having one or more comorbidities.

Comparing the results from the listwise deletion (in Table 7), the hot deck imputation in Table 8, and the multiple imputation in Table 9, all of the three methods found the relationship between the likelihood of having comorbidities and the 2 
explanatory variables (sex and geographic region). However, the multiple imputation and hot deck imputation also found a significant relationship between the likelihood of having comorbidities and the other explanatory variable age. In reality it is very reasonable that older patients would tend to have more comorbidities than younger patients. Listwise deletion, on the other hand, did not suggest age as a significant predictor variable to the likelihood of having comorbidities. There were other important differences between these results. The standard errors of the estimated regression coefficients generated by the two imputation methods, were generally smaller than those obtained by the listwise deletion method. Plausible reasons for these observed differences include the consequences of increased statistical power due to increased sample size which were resulted from applying the imputation methods.

The results based on the multiple imputation in Table 9 were very similar to the results based on the hot deck imputation in Table 8. Not only did both of the models found the same relationship between the logit of the dependent variable and the three explanatory variables (sex, age and geographic region), but also the estimated regression coefficients in the two models were very close to each other. 


\section{CONCLUSIONS AND LIMITATIONS}

\section{$\underline{\text { Conclusions }}$}

In our study, we reviewed and compared some commonly used methods for handling missing data in large surveys. Specifically, we applied three selected methods: listwise deletion, hot deck imputation, and multiple imputation in our study. The properties and effects of these methods were studied.

Our results showed differences between subjects with complete information on all variables and those whose data were incomplete. In the analysis data set generated from the 2001 NHDS, respondents with missing race data were significantly different from those with complete information with regard to the comorbidities and geographic regions.

In comparison of the three selected methods for handling missing data in our study, notable differences in the parameter estimates were seen. Both multiple imputation and hot deck imputation procedures provided more reliable parameter estimates than did listwise deletion. A similar outcome was observed with respect to the standard errors of the parameter estimates, with the multiple imputation and hot deck imputation producing parameter estimates with smaller standard errors.

With respect to the significance levels associated with the statistical tests for the parameter estimates, the multiple imputation outperformed the hot deck imputation. Multiple imputation generally yielded larger $p$ values for parameter estimates of the variables with missing values than did the hot deck imputation. This difference illustrates 
how multiple imputation captures the uncertainty with missing values.

These findings underscore the importance of employing an imputation technique rather than simply relying on complete cases for analytic purpose. Employing an imputation technique contributes to an increase in sample size and thus, an increase in statistical power. Multiple imputation even did a better job for handling missing data problem by reflecting the uncertainty associated with missing values. Furthermore, multiple imputation with the Markov Chain Monte Carlo (MCMC) method used not only the covariate information that was associated with whether the imputed values are missing but also the correlations among variables when doing imputation. Therefore, multiple imputation may preserve the relationships among variables in the regression model.

Many researchers in a variety of disciplines have stressed the need for innovative strategies to prevent, identify and handle the problem of missing data. However, few researchers utilizing the public use survey such as the NHDS have employed the imputation techniques that are currently available to handle missing data. Information regarding the similarities and discrepancies among these imputation techniques on the conclusions drawn may be useful to researchers who are currently struggling with missing data and their effects.

\section{Limitations of the Study}

There are several limitations in this study. The main limitation is that the multivariate normal model was used to impute the categorical variable race in the multiple imputation, which was actually not the true situation. In this study, the 
imputation model used for the multiple imputation contains two categorical variables: race and geographic regions, and thus do not follow a multivariate normal distribution. However, several studies have confirmed the robustness of using the multivariate normal model to impute categorical variables when these categorical outcomes are transformed into binary outcomes (Allison, 2002; Schafer, 1997). Although the multiple imputations are quite robust and still do a good job even if the assumption of multivariate normality does not hold, a more suitable model in this situation is actually the unrestricted multinomial model or a log-linear model that allows restrictions on the multinomial parameters (Schafer, 1997). However, because of the unavailability of corresponding programs in commonly used commercial software such as SAS and SPSS combined with the intensive difficulties in effective use and interpretation of these methods, they are usually not feasible in practice for many researchers.

Another limitation of this study is that only a single simulation study was conducted and reported. Additional simulations would randomly vary the respondents with missing data and vary the percentages of the missing rate. This would allow for the modeling of more realistic patterns of missingness. In addition, the distributional properties of the parameter estimates could be observed, and the performance of the method under a wide range of missing data mechanisms could also be explored. Therefore, simulation results presented in this study may differ from those if a more complete simulation effort had been undertaken.

Finally, our study only compared the performance of the three methods for handling the problem of missing data with a univariate pattern (only one variable race 
had missing data). The more realistic case of missing data with a general pattern containing several missing variables in a survey analysis remains to be explored. 


\section{REFERENCES}

Allison, PD. (2000). Multiple imputation for missing data: a cautionary tale. Sociological Methods and Research. 28:301-309.

Allison, PD. (2002). Missing Data. Thousand Oaks, CA: Sage.

Brick JM, Kalton G. (1996). Handling missing data in survey research. Statistical Methods in Medical Research. 5: 215-238.

Centers for Disease Control and Prevention. Available online at http://www.cdc.gov/nchs/about/major/hdasd/nhds.htm.

Cohen J \& Cohen P. (1983). Applied multiple regression/correlation analysis for the behavioral sciences. Hillsdale, NJ: Lawrence Erlbaum Associates.

Curran D, Bacchi M, et al. (1998). Identifying the types of missingness in quality of life data from clinical trial. Statistics in Medicine. 17(5-7): 697-709.

D'Hoore W, Sicotte C, Tilquin C. (1996). Practical considerations on the use of the Charlson Comorbidity Index with administrative sata bases. J Clin Epidemiol. 49:1429-1433

Donner, A. (1982). The relative effectiveness of procedures commonly used in multiple regression analysis for dealing with missing data. The American Statistician. 36: $378-381$

Downey RG, King CV. (1998). Missing data in likert ratings: a comparison of replacement methods. The Journal of General Psychology. 125(2): 175-191.

Fairclough DL, Peterson HF, Chang V. (1998). Why are missing quality of life data a problem in clinical trials of cancer therapy? Statistics in Medicine: 17(5-7): 667-677.

Fayers PM, Curran D, Machin D. (1998). Incomplete quality of life data in randomized trials: missing items. Statistics in Medicine. 17(5-7): 679-696.

Ford E, Cooper R, et al. (1989). Coronary arteriography and coronary bypass surgery among whites and other racial groups relative to hospital-based incidence rates for coronary artery disease: Findings from NHDS. Am J Public Health. 79(40):437-440.

Freedman VA, Wolf DA. (1995). A case study on the use of multiple imputation. 
Demography. 32:459-470.

Gergen PJ, Weiss KB. (1990). Changing patterns of asthma hospitalization among children: 1979 to 1987. JAMA. 264(13): 1688-1692.

Giles WH, Anda RF, et al. (1995). Race and sex differences in rates of invasive cardiac procedures in US hospitals. Arch Intern Med. 155(3): 318-324.

Gillum BS, Graves EJ, Wood E. (1998). National hospital discharge survey. Vital Health Stat 13. 133:1-51.

Hair JE JR, Anderson RE, Tatham RL, Black WC. (1998). Multivariate Data Analysis. Upper Saddle River, New Jersey: Prentice Hall.

Healthy People 2010. U.S. Department of Health and Human Services. (2000). (conference Edition, in two volumes). Washington, DC. http://www.health.gov/healthypeople/

Huisman M. (1999). Item nonreponse: Occurrence, causes, and imputation of missing answers to test items. Leidan, the Netherlands: DSWO Press.

King G, Honaker J, et al. (2001). Analyzing incomplete political science data: an alternative algorithm for multiple imputation. American Political Science Review. 95(1): 49-69.

Kline, R.B. (1998). Principles and practice of structural equation modeling. New York: Guilford.

Kozak LJ. (1995). Underreorting of race in the National Hospital Discharge Surveys. Adv Data. 265: 1-12.

Kozak LJ, McCarthy E, et al. (1993). Patterns of hospitals use by patients with diagnoses related with HIV infection. Public Health Rep. 108(5): 571-581.

Lavori PW, Dawson R, and Shera D. (1995). Amultiple imputation strategy for clinical trials with truncation of patient data. Statistics in Medicine. 14:1913-1925.

Levy PS, Lemeshow S. (1991). Sampling of Populations: Methods and Applications. John Wiley \& Sons, INC.

Little, RJA and Rubin DB. (1987). Statistical Analysis with Missing Data. John Wiley \& Sons, New York.

Little, RJA and Rubin DB. (2002). Statistical Analysis with Missing Data. John Wiley \& Sons, New York. 
Livingston EH, Huerta S, et al. (2002). Male gender is a predictor of morbidity and age a predictor of mortality for patients undergoing gastric bypass surgery. Ann Surg. 236: 576-582.

McNally JW. (1997). Generating hot-deck imputation estimates: using SAS for simple and multiple imputation allocation routines. Brown University. PSTC Working Paper. \#97-12.

Modan B, Wagener DK, et al. (1992). Some epidemiological aspects of stroke: Mortality/morbidity trends, age, sex, race, socioeconomic status. Stroke. 23(9): 1230-1236.

Mokdad AH, Bowman BA, Ford ES, et al. (2000). The continuing epidemic of obesity in The United States. JAMA. 284:1650-1651

Mun EC, Blackburn GL, Matthews JB. (2001). Current status of medical and surgical therapy for obesity. Gatroenterology. 120: 669-681.

Musil CM, Warner CB, Yobas PK, et al. (2002). A comparison of imputation techniques for handling missing data. Western Journal of Nursing Research. 24(7):815-829.

Patrician P.A. (2002). Focus on research methods multiple imputation for missing data. Research in Nursing \& Health. 25: 76-84.

Perez A, Rodolfo JD, et al. (2002). Use of the mean, hot deck and multiple imputational techniques to predict outcome in intensive care unit patients in Colombia. Statistics in Medicine. 21:3885-3896.

Pope GD, Birkmeyer JD, Finlayson SRG. (2002). National trends in utilization and inhospital outcomes of bariatric surgery. J Gastrointest Surg. 6: 855-860.

Rand CSW, Kuldau JM. (1993). Morbid obesity-a comparison between a generalpopulation and obesity surgery patients. Int J Obes Relat Metab Disord. 17: 657-661.

Reilly M \& Pepe M. (1997). The relationship between hot-deck multiple imputation and weighted likelihood. Statistics in Medicine. 16: 5-19.

Rodriguez JG, Sattin RW, et al. (1989). Incidence of hip fractures, United States, 197083. Am J Prev Med. 5(3): 175-181.

Romano PS, Roos LL, Jollis JG. (1993). Adapting a clinical comorbidity index for use with ICD-9-CM administrative data-differing perspectives. J Clin Epidemiol. 46: 1075-1079.

Rosenbaum PR, Rubin DB. (1987). The central role of the propencity score in 
observational studies for causal effects. Biometrika. 70: 41-55.

Rubin DB. (1976). Inference and missing data. Biometrics, 63: 581-592.

Rubin DB. (1978). Multiple imputations in sample surveys-a phenomenological Bayesian approach to non-response. Proceedings of the Survey Research Methods Section. American Statistical Association. 20-34

Rubin DB. (1987). Multiple imputation for nonresponse in surveys. New York: John Wiley.

Rubin DB. (1996). Multiple imputation after 18+ years. Journal of the American Statistical Association. 91: 473-515

SAS Institute Inc. (1999). SAS Procedures Guide, Version 8. Cary, NC: SAS Institute Inc.

Schafer JL. (1997). Analysis of incomplete multivariate data. New York: Chapman \& Hall.

Schafer JL, Olsen MK. (1998). Multiple imputation for multivariate missing data problems: A data analyst's perspective. Multivariate Behavior Research. 33: 545571

Schafer JL. (1999). Multiple imputation: a primer. Statistical Methods in Medical Research. 8: 3-15.

Schenker N, Parker JD. (2003). From single-race reporting to multiple-race reporting: using imputation methods to bridge the transition. Statistics in Medicine. 22: 15711587.

Tabachnick BG \& Fridell LS. (2001). Using multivariate statistics. Needham Heights, MA: Allyn \& Bacon.

Troxel AB, Fairclough SV, et al. (1998) Statistical analysis of quality of life with missing data in cancer clinical trials. Statistics in Medicine. 17(5-7):653-666.

Van Buuren, S \& Oudshoorn K. (2001). Multiple Imputation Online. TNO Prevention and Health, Department of Statistics. http://www.multipleimputation.com

Waetijen LE, Subak LL, Shen H, et al. (2003). Stress urinary incontinence surgery in the United States. Obstetrics \& Gynecology. 101(4): 1177-1182.

Weber AM, Meyn L. (2002). Episiotomy use in the United States, 1979-1997. Obstetrics \& Gynecology. 100(6): 671-676

Wilcox LS, Kooin LM, et al. (1994). Hysterectomy in the United States, 1988-1990. 
Obstet-Gynecol. 83(4): 549-555.

Yang CY. (2000). Multiple imputation for missing data: concepts and new development. SAS Institute Inc.

Zizza CA, Herring AH, Stevens J, et al. (2003). Bariatric surgeries in North Carolina, 1990 to 2001: A gender comparison. Obesity Research. 11(12): 1519-1527. 


\section{APPENDIX}



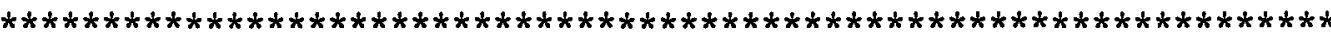

$\star * * * * *$

options ls=78;

libname missing ' $\mathrm{C}: \mathrm{l}^{\prime}$;

$\%$ let bar=simulation;

$\%$ let key=race;

$\%$ let ID=order;

*list the variables in the donor pool to be used for the hot deck;

\%let Donpool = \&key flag age_grp sex geo cci;

* list the variables in the recipient pool to be used for hot deck

after renaming them with the m suffix: ie. age=mage;

\%let Getpool= \&key \&Id flag mrace age_grp mage_grp sex msex geo mgeo cci mcci; 
$* * *$ retreive the donor pool $* * *$;

data donor;

set missing.\&bar;

keep \&donpool;

if \&key ne . ; *value to denote missing case;

Flag=1;

proc print;

title'donor pool';

run;

*** retreive the receiving pool ***;

data $\operatorname{missing}($ rename $=($ age grp $=$ mage_grp $\operatorname{sex}=$ msex $g e o=$ mgeo race $=$ mrace cci $=$ mcci $)$;

set missing.\&bar;

keep \&getpool;

if \&key eq .; *. to denote missing case*;

Flag=1;

proc print;

title'missing pool';

run;

proc means noprint;

var flag;

output out $=$ miss sum=total;

data missing.report;

set missing;

keep \&id;

proc print;

run;

proc sort data=missing.report;

by order;

run;

*** set the number of iterations ***;

\%global m k Fuzz1 missl donate1 replace;

Data Num;

Set miss end=last;

format total 1.0;

Flag=1;

$\mathrm{jm}=$ FREQ_;

if flag $=1$ then $\mathrm{jm}+0$;

if last then call symput ('m', left(jm));

$\%$ let $\mathrm{k}=1$; * number of imputation rounds;

$\%$ let Fuzz1 $=0$; *amount of variation in matches;

$\%$ let Fuzz2 $=0$; *amount of variations in matches;

\%let Fuzz3 $=0$; *amount of variations in matches;

\%let Fuzz4 $=0$; *amount of variations in matches;

$\%$ let miss1 = mage_grp ; *matching variable from miss for hot deck; 
$\%$ let donate1 = age_grp ; *matching variables from donor for hot deck;

$\%$ let replace $=$ race; *variable to be hotdecked into data set;

$\%$ let miss $2=$ msex ; * matching variable from miss for hot deck;

$\%$ let donate $2=$ sex; *matching variable from donor for hot deck;

$\%$ let miss $3=$ mgeo; *matching variable from miss for hot deck;

$\%$ let donate $3=$ geo; ${ }^{*}$ matching variable from donor for hot deck;

$\%$ let miss $4=$ mcci; * matching variable from miss for hot deck;

$\%$ let donate4= cci; ${ }^{*}$ matching variable from donor for hot deck;

$* * * *$ perform the macro function ${ }^{* * * * *}$;

$\%$ macro create(a);

$\%$ do \%until (\&a gt \&m);

$\%$ do $\mathrm{i}=1 \%$ to $\&$ k \%by 1 ;

Data two;

set missing;

if_n_=\&a;

data three;

merge Donor two;

by flag;

if $\&$ miss 1 ne \&donate 1 then delete;

if \&miss 2 ne \&donate2 then delete;

if \&miss 3 ne \&donate 3 then delete;

if \&miss 4 ne \&donate4 then delete;

pick=ranuni $(\mathbf{0})$;

proc sort;

by pick;

data four;

set three;

if_n_ $=1$;

imp \&i=\&replace;

keep \&id imp\&i;

data missing.report;

merge missing.report four;

by order;

$\%$ end;

$\%$ if i lt \&k \%then \%let a $=\%$ eval( $\& a)$;

$\%$ else \%let a $=\%$ eval $(\& a+1)$;

$\%$ end;

$\%$ mend create;

$\%$ create (1);

run;

data data.simu_race;

set missing.report;

run;

data data.simu_donor;

set donor; 
run;

data data.simu_missing;

set missing;

run;

proc sort data=data.simu_race;

by order;

run;

proc sort data $=$ data.simulation;

by order;

run;

data data.simu_merge;

merge data.simu_race data.simulation;

by order;

if race $=$. then race $=\mathrm{impl}$;

run;

$* * * * * * * * * * *$ logistic regression for hot-deck imputed data

$* * * * * * * *$

proc logistic data=data.simu_merge descending;

class race geo /ref=first;

model $\mathrm{cci}=$ sex age race geo;

weight wgt;

run;

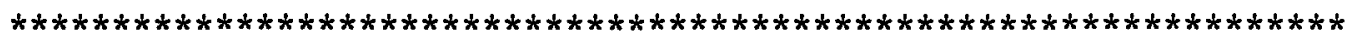

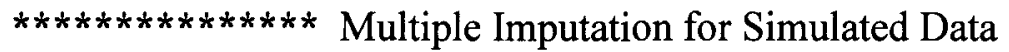

$* * * * * * * * * * * * * * *$

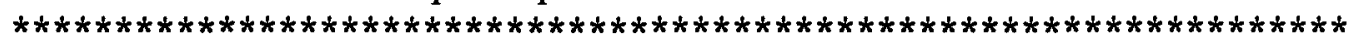

proc mi data $=$ simulation seed $=123$ out $=$ simu_mi nimpute $=5$

round $=0.01$ minimum $=0$ maximum $=1$;

mcmc chain $=$ multiple nbiter $=200$ displayinit initial $=\mathrm{em}$ (itprint)

timeplot(wlf) acfplot (wlf);

var cci sex age geol geo2 geo3 race1 race $2 \mathrm{wgt}$;

run;

data data.simu_mi;

set simu_mi;

race3 $=1$-race1-race2;

run;

data data.simu_mi;

set data.simu_mi;

if race $1=\max ($ race 1 ,race 2 ,race 3$)$ then race $m i=2$;

else if race $2=\max ($ race 1, race 2 ,race 3$)$ then race $m i=3$;

else race $\mathrm{mi}=\mathbf{1}$;

run; 
$* * * * * * * * * *$ Logistic Regression for the 5 Imputed Data Sets $* * * * * * * * * * * * *$ proc logistic data=data.simu_mi outest $=$ outlogit_simu_mi covout descending; class race_mi geo /param=ref; model $\mathrm{cci}=$ sex age race_mi geo; weight wgt; by_imputation_;

run;

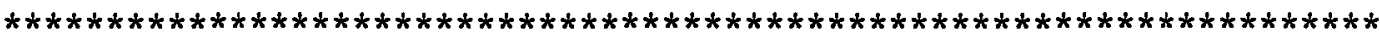
$* * * * * * * * * * * * * *$ Proc Mianalyze to Pool the Results from the $* * * * * * * * * * *$

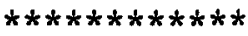
5 Imputed Data Sets to Obtain a Final Result $* * * * * * * * * * *$ $* * * * * * * * * * * * * * * * * * * * * * * * * * * * * * * * * * * * * * * * * * * * * * * * * * * * * * * * * * * * * * * * * * *$

proc mianalyze data $=$ outlogit_simu_mi edf $=274$;

var intercept sex age race_miblack race_miother geomidwest geonortheast geosouth;

run; 


\section{CURRICULUM VITAE}

NAME: $\quad$ Ping Xu

ADDRESS: $\quad$ Department of Bioinformatics and Biostatistics

555 S. Floyd Street

University of Louisville

Louisville, KY 40202

DOB:

Tengzhou, Shandong, P.R. China - Feb 2, 1975

EDUCATION

\& TRAINING: $\quad$ B.S., Pediatrics

Qingdao University, China

1993-98

M.S., Pediatrics

Capital University of Medical Sciences, China

1998-2001

MSPH, Biostatistics-Decision Science

University of Louisville

2002-2004

PROFESSIONAL

SOCIETIES:

American Statistical Association

PUBLICATIONS: Ping Xu, Ding Zongyi. Progress of research on apoptosis of adipocytes (review). Chinese Journal of Pediatrics; 2000, 38(1):58-59.

INVITED

PRESENTATIONS: Ping Xu, Tonya M. Smoot. Gastric bypass surgery in the United States, 2000-2001. Research Louisville, University of Louisville. 2003 Nov (Poster Presentation).

Ping Xu, Ding Zongyi, Du Lirong. Study on basal apoptosis of adipocytes in Chinese obese and non-obese children. The $23^{\text {rd }}$ International Congress of Pediatrics; 2001 Sep (Oral Presentation). 